\title{
ON THE SEMI-GLOBAL STABILIZABILITY OF THE KORTEWEG-DE VRIES EQUATION VIA MODEL PREDICTIVE CONTROL *
}

\author{
BEHZAD AZMI ${ }^{1}$, ANNE-CÉLINE BOULANGER ${ }^{2}$ AND KARL KUNISCH ${ }^{3}$
}

\begin{abstract}
Stabilization of the nonlinear Korteweg-de Vries (KdV) equation on a bounded interval by model predictive control (MPC) is investigated. This MPC strategy does not need any terminal cost or terminal constraint to guarantee the stability. The semi-global stabilizability is the key condition. Based on this condition, the suboptimality and exponential stability of the model predictive control are investigated. Finally, numerical experiment is presented which validates the theoretical results.
\end{abstract}

1991 Mathematics Subject Classification. 49N35, 93C20, 93D20.

The dates will be set by the publisher.

\section{INTRODUCTION}

The present work is devoted to model predictive control in the context of stabilization of the nonlinear Korteweg-de Vries equation

$$
\partial_{t} y+\partial_{x} y+y \partial_{x} y+\partial_{x}^{3} y=0
$$

where $y=y(t, x)$ is a real valued function of real variables $t$ and $x$. The $\mathrm{KdV}$ equation was first derived by Boussinesq 12 and rediscovered by Korteweg and de Vries [37 as a model for the propagation of water waves along a channel. This equation serves also as a very useful approximation in studies aiming to include and balance a weak nonlinearity and weak dispersive effects. Particularly, the equation is now commonly used as a mathematical model for the unidirectional propagation of small amplitude long waves in nonlinear dispersive systems. In the past decades, many authors studied the KdV equation from various aspects of mathematics, including the well-posedness, existence and stability of solitary waves, the long-time behavior, stabilization, and the controllability. Among all of them we can point out the

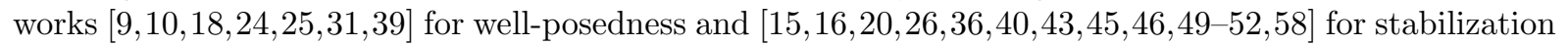
and control theory.

Here we consider the following optimal control problem which consists of minimizing the performance index

$$
J_{\infty}\left(u, y_{0}\right):=\int_{0}^{\infty} \ell(y(t), u(t)) d t
$$

Keywords and phrases: receding horizon control, model predictive control, asymptotic stability, infinite-dimensional systems

* This work has been supported by the International Research Training Group IGDK1754, funded by the DFG and $F W F$, and the ERC advanced grant 668998 (OCLOC) under the EU's H2020 research program.

${ }^{1}$ Institute for Mathematics and Scientific Computing, University of Graz, Heinrichstraße 36, 8010 Graz, Austria e-mail: behzad.azmi@uni-graz.at

2 e-mail: anneceline.boulanger@gmail.com

${ }^{3}$ Institute for Mathematics and Scientific Computing, University of Graz, Heinrichstraße 36, 8010 Graz, Austria e-mail: karl.kunisch@uni-graz.at and Radon Institute, Austrian Academy of Sciences

(c) EDP Sciences, SMAI 1999 
subject to the Korteweg-de Vries $(\mathrm{KdV})$ equation posed on the space-time cylinder $(0, L) \times[0, \infty)$

$$
\begin{cases}\partial_{t} y+\partial_{x} y+y \partial_{x} y+\partial_{x}^{3} y=B u & x \in(0, L), \quad t>0 \\ y(t, 0)=y(t, L)=\partial_{x} y(t, L)=0 & t>0 \\ y(0, \cdot)=y_{0} & x \in(0, L)\end{cases}
$$

where the external control $u(t)=u(t, x)$ is real valued function, and $y_{0} \in L^{2}(0, L)$. The control operator $B$ is the extension-by-zero operator given by

$$
(B u)(x)= \begin{cases}u(x) & x \in \hat{\Omega}, \\ 0 & x \in(0, L) \backslash \hat{\Omega},\end{cases}
$$

where the control domain $\hat{\Omega}$ is a nonempty open subset of $(0, L)$. Further, the incremental function $\ell: L^{2}(0, L) \times L^{2}(\hat{\Omega}) \rightarrow \mathbb{R}^{+}$is defined by

$$
\ell(y, u):=\frac{1}{2}\|y\|_{L^{2}(0, L)}^{2}+\frac{\beta}{2}\|u\|_{L^{2}(\hat{\Omega})}^{2} .
$$

One strategy to deal with problem (1)-(2) is model predictive control (MPC) which is also known as the receding horizon control approach. In this strategy a suboptimal solution of (1)-(2) is obtained: a sequence of finite horizon optimal control problems on a family of finite horizon intervals covering $[0, \infty)$ is solved, and these locally optimal controls are concatenated to a subopitmal control on $[0, \infty)$. The asymptotic stability of the resulting controlled system is not a-priori guaranteed. It can even be demonstrated by a simple linear example that the naive application of a model predictive control strategy can lead to an unstable controlled system. Thus, often it is necessary to impose additional conditions or add terminal costs or terminal constraints to the finite horizon problems to guarantee the desired system performance.

In the past three decades, numerous results have been published on model predictive control for finitedimensional systems $1,2,17,27,29,30,34,35,44$ and the many references therein. Recently the case of infinite-dimensional systems was considered as well 28, 32, 33. In [32, a general framework to stabilize infinite-dimensional dynamical systems was introduced. In this framework the stability of the controlled system is ensured by adding control Lyapunov functions as terminal cost to the finite horizon problems. More recently several authors, see e.g [27, 28, 30, 34] managed to prove the asymptotic stabilizability of MPC even without use of control Lyapunov functions and terminal constraints. This class of MPC strategies is called "unconstrained" MPC strategies 28. So far, this class of strategies has been well studied for finite-dimensional dynamical systems [34, 48] and discrete time dynamical systems [27,30. But, there are only few results for infinite-dimensional systems with continuous time dynamics. In the present work we continue our study, initiated in [4], on the analysis of the unconstrained MPC strategy for infinite-dimensional controlled systems. Depending on the type of boundary conditions the suboptimality and local, respectively, global asymptotic stability of the MPC controls were verified for the Burgers' equation. In this paper, based on the semi-global stabilizability result from [46] we first show that the MPC control for (2) is suboptimal. Then by an observability type estimate, we prove that the resulting MPC-controlled system is semi-globally exponentially stable. This requires techniques which differ from those which were employed in [4].

To briefly explain the model predictive control approach, we choose a sampling time $\delta>0$ and a prediction horizon $T>\delta$. Then sampling instances $t_{k}:=k \delta$ for $k=0,1, \ldots$ are defined. At every sampling instance $t_{k}$, an open-loop optimal control problem is solved over the finite prediction horizon $\left[t_{k}, t_{k}+T\right]$. The optimal control thus obtained is applied to steer the system from time $t_{k}$ with the initial state $y_{m p c}\left(t_{k}\right)$ until time $t_{k+1}:=t_{k}+\delta$ at which point, a new measurement of state is assumed to be available. The process is repeated starting from the new state: we obtain a new optimal control and a new predicted state trajectory by shifting the prediction horizon forward in time. Throughout, we denote the model predictive state- and control variables by $y_{m p c}(\cdot)$ and $u_{m p c}(\cdot)$, respectively. Also, 
$\left(y_{T}^{*}\left(\cdot ; y_{0}, t_{0}\right), u_{T}^{*}\left(\cdot ; y_{0}, t_{0}\right)\right)$ stands for the optimal state and control of the optimal control problem with finite time horizon $T$, and initial function $y_{0}$ at initial time $t_{0}$. This is summarized in Algorithm 1 .

The remainder of this paper is organized as follows: Section 2 deals with global well-posedness of the nonlinear KdV equation in the weak sense. In Section 3, existence of the finite horizon optimal control is investigated. Section 4 analyzes the suboptimality and semi-global exponential stability of the model predictive control obtained by Algorithm 1. Finally, Section 5 is devoted to numerical simulations.

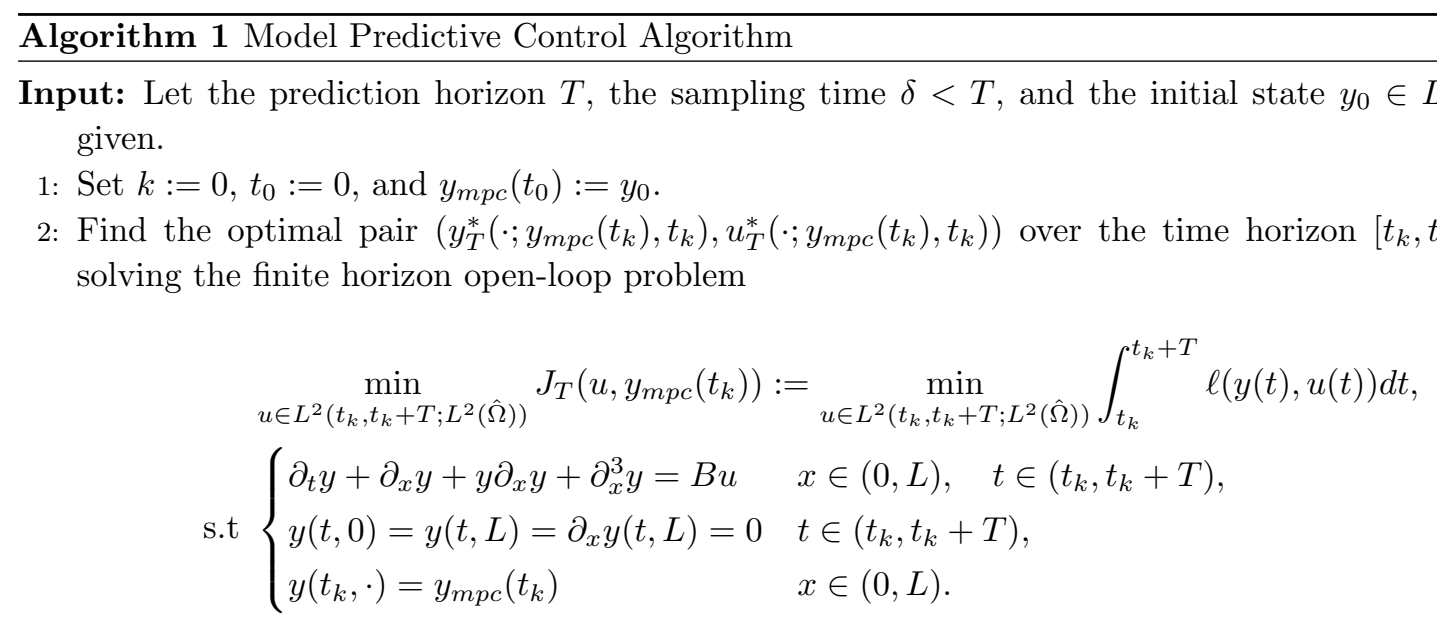

3: Set

$$
\begin{aligned}
u_{m p c}(\tau) & :=u_{T}^{*}\left(\tau ; y_{m p c}\left(t_{k}\right), t_{k}\right) \quad \text { for all } \tau \in\left[t_{k}, t_{k}+\delta\right), \\
y_{m p c}(\tau) & :=y_{T}^{*}\left(\tau ; y_{m p c}\left(t_{k}\right), t_{k}\right) \quad \text { for all } \tau \in\left[t_{k}, t_{k}+\delta\right], \\
t_{k+1} & :=t_{k}+\delta, \\
k & :=k+1 .
\end{aligned}
$$

4: Go to Step 2.

\section{Well-POSEDNESS OF THE KDV EQUATION}

In this section we deal with the existence of global solution of the nonlinear KdV equation

$$
\begin{cases}\partial_{t} y+\partial_{x} y+\partial_{x}^{3} y+y \partial_{x} y=f & x \in(0, L), \quad t \in(0, T), \\ y(t, 0)=y(t, L)=\partial_{x} y(t, L)=0 & t \in(0, T), \\ y(0, \cdot)=y_{0} & x \in(0, L),\end{cases}
$$

with an arbitrary finite time horizon $T$, forcing function $f \in L^{2}\left(0, T ; L^{2}(0, L)\right)$, and initial function $y_{0} \in L^{2}(0, L)$. Throughout we shall refer to the following function spaces:

$$
\mathcal{B}_{0, T}:=C\left([0, T] ; L^{2}(0, T)\right) \cap L^{2}\left(0, T ; H_{0}^{1}(0, L)\right)
$$

equipped with the norm

$$
\|v\|_{\mathcal{B}_{0, T}}:=\sup _{t \in[0, T]}\|v(t)\|_{L^{2}(0, L)}+\|v\|_{L^{2}\left(0, T ; H_{0}^{1}(0, L)\right)},
$$


the space in which solutions will be sought

$$
\mathcal{W}_{0, T}:=L^{2}\left(0, T ; H_{0}^{1}(0, L)\right) \cap C\left([0, T] ; L^{2}(0, L)\right) \cap H^{1}\left(0, T ; H^{-2}(0, L)\right),
$$

and the space of test functions

$$
\mathcal{X}:=\left\{\omega \in H^{2}(0, L) \mid \omega(0)=\omega(L)=\omega^{\prime}(0)=0\right\} .
$$

First of all we recall that, for every forcing function $f \in L^{1}\left(0, T ; L^{2}(0, L)\right)$ and initial function $y_{0} \in$ $L^{2}(0, L)$, the linear $\mathrm{KdV}$ equation

$$
\begin{cases}\partial_{t} y+\partial_{x} y+\partial_{x}^{3} y=f & x \in(0, L), \quad t \in(0, T) \\ y(t, 0)=y(t, L)=\partial_{x} y(t, L)=0 & t \in(0, T) \\ y(0, \cdot)=y_{0} & x \in(0, L)\end{cases}
$$

is well-posed. We consider the operator $\mathcal{A}:=-\partial_{x}-\partial_{x}^{3}$ on the dense domain $\mathcal{D}(\mathcal{A}) \subset L^{2}(0, L)$ defined by

$$
\mathcal{D}(\mathcal{A}):=\left\{\phi \in H^{3}(0, L) \mid \phi(0)=\phi(L)=\phi^{\prime}(L)=0\right\} .
$$

It has been shown [49] that $\mathcal{A}$ and its adjoint $\mathcal{A}^{*}$ with domain

$$
\mathcal{D}\left(\mathcal{A}^{*}\right):=\left\{\phi \in H^{3}(0, L) \mid \phi(0)=\phi(L)=\phi^{\prime}(0)=0\right\} .
$$

are dissipative. Therefore, due to 47] (cor. 4.4 Chapter 1 Page 15) the operator $\mathcal{A}$ is the infinitesimal generator of a $C_{0}$-semigroup of contractions $\{W(t)\}_{t \geq 0}$ on $L^{2}(0, L)$ and we have the mild form of the solution to (6) given by

$$
y(t)=W(t) y_{0}+\int_{0}^{t} W(t-s) f(s) d s \quad \text { for all } t \in[0, T] .
$$

Moreover, we have the following result for this mild solution from [49].

Theorem 1.1. Let $T, L>0$ be given. For any $y_{0} \in L^{2}(0, L)$ and any $f \in L^{1}\left(0, T ; L^{2}(0, L)\right)$, the Cauchy problem (6) admits a unique mild solution which belongs to the space $\mathcal{B}_{0, T}$. Furthermore, for the mild solution $y$ we have the estimate

$$
|y|_{L^{2}\left(0, T ; H_{0}^{1}(0, T)\right)}+\sup _{0 \leq t \leq T}|y(t)|_{L^{2}(0, L)} \leq C\left(\left|y_{0}\right|_{L^{2}(0, L)}+|f|_{L^{1}\left(0, T ; L^{2}(0, L)\right)}\right),
$$

where the constant $C>0$ depends on $L$ and $T$.

Lemma 1.1. Let $T>0$ and $y \in \mathcal{B}_{0, T}$. Then $y \partial_{x} y \in L^{1}\left(0, T ; L^{2}(0, L)\right)$. Moreover the mapping $y \in$ $\mathcal{B}_{0, T} \rightarrow y \partial_{x} y \in L^{1}\left(0, T ; L^{2}(0, L)\right)$ is continuous, and for every $y, z \in \mathcal{B}_{0, T}$ we have the following estimate

$$
\left\|y \partial_{x} y-z \partial_{x} z\right\|_{L^{1}\left(0, T ; L^{2}(0, L)\right)}=C_{a} T^{\frac{1}{4}}\left(\|y\|_{\mathcal{B}_{0, T}}+\|z\|_{\mathcal{B}_{0, T}}\right)\|y-z\|_{\mathcal{B}_{0, T}}
$$

where $C_{a}$ is a positive constant independent of $T$.

Proof. The proof is given in Appendix A.1.

We now turn to the nonlinear equation.

Definition 1.1 (Mild solution). Suppose that $T>0$ is arbitrary, and we are given $f \in L^{1}\left(0, T ; L^{2}(0, L)\right)$ and $y_{0} \in L^{2}(0, L)$. Then $y \in \mathcal{B}_{0, T}$ is referred to as a mild solution to (4) if the following integral equation is satisfied

$$
y(t)=W(t) y_{0}-\int_{0}^{t} W(t-s)\left(y \partial_{x} y\right)(s) d s+\int_{0}^{t} W(t-s) f(s) d s \quad \text { for all } t \in[0, T],
$$


where the $C_{0}$-semigroup of contractions $\{W(t)\}_{t \geq 0}$ defined in the above for the linear KdV equation (6).

Theorem 1.2. Let $T, L>0$ be given. For any $y_{0} \in L^{2}(0, L)$ and $f \in L^{1}\left(0, T ; L^{2}(0, L)\right)$, there exists a $T^{*} \in[0, T]$ depending on $\left|y_{0}\right|_{L^{2}(0, L)}$ and $|f|_{L^{1}\left(0, T ; L^{2}(0, L)\right)}$ such that (4) admits a unique solution in the space $\mathcal{B}_{0, T^{*}}$.

Proof. We express problem (4) as a fixed point equation $y=\Psi(y)$. For this purpose we write (4) in integral form as

$$
y(t)=W(t) y_{0}-\int_{0}^{t} W(t-s)\left(y \partial_{x} y\right)(s) d s+\int_{0}^{t} W(t-s) f(s) d s .
$$

For any $r>0$ and time horizon $\theta$ we define the ball $S_{\theta, r}$ centered at zero by $S_{\theta, r}:=\left\{x \in \mathcal{B}_{0, \theta},|x|_{\mathcal{B}_{0, \theta}} \leq r\right\}$. This is a closed, convex, and bounded subset of $\mathcal{B}_{0, \theta}$. We define the mapping $\Psi$ on $S_{\theta, r}$ by

$$
\Psi(y):=W(t) y_{0}-\int_{0}^{t} W(t-s)\left(y \partial_{x} y\right)(s) d s+\int_{0}^{t} W(t-s) f(s) d s \quad \text { for } y \in S_{\theta, r} .
$$

Then by (7) and (8), we have

$$
\begin{aligned}
|\Psi(y)|_{B_{0, \theta}} & \leq C\left(\left|y_{0}\right|_{L^{2}(0, L)}+|f|_{L^{1}\left(0, T ; L^{2}(0, L)\right)}+\left|y \partial_{x} y\right|_{L^{1}\left(0, T ; L^{2}(0, L)\right)}\right) \\
& \leq C\left(\left|y_{0}\right|_{L^{2}(0, L)}+|f|_{L^{1}\left(0, T ; L^{2}(0, L)\right)}\right)+C C_{a} \theta^{\frac{1}{4}}|y|_{B_{0, \theta}}^{2} .
\end{aligned}
$$

Choosing the $r$ and $\theta$ such that

$$
\left\{\begin{array}{l}
r=\frac{4}{3} C\left(\left|y_{0}\right|_{L^{2}(0, L)}+|f|_{L^{1}\left(0, T ; L^{2}(0, L)\right)}\right), \\
C C_{a} \theta^{\frac{1}{4}} r \leq \frac{1}{4} .
\end{array}\right.
$$

we obtain

and

$$
|\Psi(y)|_{B_{0, \theta}} \leq r \quad \text { for all } y \in S_{\theta, r}
$$

$$
\left|\Psi\left(y_{1}\right)-\Psi\left(y_{2}\right)\right|_{B_{0, \theta}} \leq \frac{1}{2}\left|y_{1}-y_{2}\right|_{B_{0, \theta}} \quad \text { for all } y_{1}, y_{2} \in S_{\theta, r}
$$

The existence of a unique solution to the Cauchy problem (4) follows by Banach's fixed point theorem. Note that by $(9)$ we have

$$
T^{*} \leq \frac{1}{\left(\frac{16 C_{a} C^{2}}{3}\left(\left|y_{0}\right|_{L^{2}(0, L)}+|f|_{L^{1}\left(0, T ; L^{2}(0, L)\right)}\right)\right)^{4}} .
$$

Therefore for any $y_{0} \in L^{2}(0, L)$ and $f \in L^{1}\left(0, T ; L^{2}(0, L)\right)$, there exists $T^{*} \in[0, T]$ depending on $\left|y_{0}\right|_{L^{2}(0, L)}$ and $|f|_{L^{1}\left(0, T ; L^{2}(0, L)\right)}$ such that (4) admits a unique solution in the space $\mathcal{B}_{0, T^{*}}$.

To show global well-posedness we need an a-priori estimate for solutions of (4). This is attained next.

Lemma 1.2. Let $T>0$ be arbitrary. Then for every $y_{0} \in L^{2}(0, L)$ and $f \in L^{2}\left(0, T ; L^{2}(0, L)\right)$, the solution $y \in \mathcal{B}_{0, T^{\prime}}$ to (4) with $T^{\prime} \in(0, T]$ satisfies the following estimate

$$
|y|_{\mathcal{B}_{0, T^{\prime}}} \leq K_{1}\left(T, L, y_{0}, f\right) .
$$

Moreover, the solution y belongs to the space $\mathcal{W}_{0, T^{\prime}}$ and we have that following estimate

$$
|y|_{\mathcal{B}_{0, T^{\prime}}}+\left|\partial_{t} y\right|_{L^{2}\left(0, T^{\prime} ; H^{-2}(0, L)\right)} \leq K_{2}\left(T, L, y_{0}, f\right),
$$

where the constants $K_{1}$ and $K_{2}$ depend on the quantities $T, L,\left|y_{0}\right|_{L^{2}(0, L)}$, and $|f|_{L^{2}\left(0, T ; L^{2}(0, L)\right)}$. Further these constants will grow unboundedly as at least one of the above quantities tends to infinity. 
Proof. The proof is given in Appendix A.2.

Theorem 1.3. Let an arbitrary $T>0$ be given. Then for every $y_{0} \in L^{2}(0, L)$ and $f \in L^{2}\left(0, T ; L^{2}(0, L)\right)$, there exists a unique mild solution $y \in \mathcal{W}_{0, T}$ for the nonlinear KdV equation (4).

Proof. Local existence due to Theorem 1.2 together with the a-priori bound 10 of Lemma 1.2 imply global existence by the standard continuation argument. Uniqueness follows from Theorem 1.2 as well.

We will later use the following useful lemma from [14 Page. 45.

Lemma 1.3. Let $E$ and $F$ be two Banach spaces and $\mathcal{A}: E \supset \mathcal{D}(A) \rightarrow F$ be a densely defined unbounded linear operator, then the adjoint operator $\mathcal{A}^{*}$ is closed. That is, the graph of this operator $\mathcal{G}\left(\mathcal{A}^{*}\right)$ is closed in $F^{*} \times E^{*}$. Moreover we have

$$
\mathcal{I}\left(\mathcal{G}\left(\mathcal{A}^{*}\right)\right)=\mathcal{G}(\mathcal{A})^{\perp}
$$

where the isomorphism $\mathcal{I}: F^{*} \times E^{*} \rightarrow E^{*} \times F^{*}$ is defined by

$$
\mathcal{I}(X, Y)=(-Y, X) \quad \text { for all }(X, Y) \in F^{*} \times E^{*} .
$$

Definition 1.2 (Weak solution). Suppose that $T>0$ is arbitrary, and we are given $f \in L^{2}\left(0, T ; L^{2}(0, L)\right)$ and $y_{0} \in L^{2}(0, L)$. Then $y \in \mathcal{W}_{0, T}$ is referred to as a weak solution to (4) if $y(0)=y_{0}$ in $L^{2}(0, L)$ and the following equality holds

$$
\left\langle\partial_{t} y(t), \phi\right\rangle_{H^{-2}, H^{2}}+\left\langle\partial_{x} y(t), \phi\right\rangle_{L^{2}}+\left\langle y(t) \partial_{x} y(t), \phi\right\rangle_{L^{2}}+\left\langle\partial_{x} y(t), \partial_{x}^{2} \phi\right\rangle_{L^{2}}=\langle f(t), \phi\rangle_{L^{2}}
$$

for almost every $t \in(0, T)$ and every $\phi \in \mathcal{X}$.

Theorem 1.4. For every $T>0, f \in L^{2}\left(0, T ; L^{2}(0, L)\right)$, and $y_{0} \in L^{2}(0, L)$, problem (4) admits a unique weak solution.

Proof. Inspired by [5.7, we first show that any mild solution of (4) is a weak solution. Let $y \in \mathcal{W}_{0, T}$ be a mild solution of (4). Then for every $t \in[0, T]$ we have

$$
y(t)=W(t) y_{0}-\int_{0}^{t} W(t-s)\left(y \partial_{x} y\right)(s) d s+\int_{0}^{t} W(t-s) f(s) d s,
$$

where $y \in C\left([0, T] ; L^{2}(0, L)\right) \subset L^{1}\left(0, T ; L^{2}(0, L)\right)$. For every $\phi \in D\left(\mathcal{A}^{*}\right)$ and $\sigma \in \mathcal{D}(0, T)$, the vectorial distributional derivative of $y$ is obtained by

$$
\begin{aligned}
-\int_{0}^{T} & \langle y(t), \phi\rangle \sigma^{\prime}(t) d t \\
& =-\int_{0}^{T}\left[\left\langle W(t) y_{0}+\int_{0}^{t} W(t-s)\left(f(s)-y(s) \partial_{x} y(s) d s, \phi\right\rangle\right] \sigma^{\prime}(t) d t\right. \\
& =-\int_{0}^{T}\left\langle W(t) y_{0}, \phi\right\rangle \sigma^{\prime}(t) d t-\int_{0}^{T} \int_{s}^{T}\left\langle W(t-s)\left(f(s)-y(s) \partial_{x} y(s)\right), \phi\right\rangle \sigma^{\prime}(t) d t d s .
\end{aligned}
$$

For every $\psi \in D(\mathcal{A})$ and $\phi \in D\left(\mathcal{A}^{*}\right)$, we can write for almost every $t>0$

$$
\frac{d}{d t}\langle W(t) \psi, \phi\rangle=\langle\mathcal{A} W(t) \psi, \phi\rangle=\left\langle W(t) \psi, \mathcal{A}^{*} \phi\right\rangle .
$$

Since $\mathcal{D}(\mathcal{A})$ is dense in $L^{2}(0, L)$, this equality can be extended for every $\psi \in L^{2}(0, T)$. Moreover, by integrating by parts we have

$$
-\int_{0}^{T}\left\langle W(t) y_{0}, \phi\right\rangle \sigma^{\prime}(t) d t=\int_{0}^{T}\left\langle W(t) y_{0}, \mathcal{A}^{*} \phi\right\rangle \sigma(t) d t
$$


and,

$$
\begin{aligned}
-\int_{s}^{T}\langle W(t-s) & \left(f(s)-y(s) \partial_{x} y(s), \phi\right\rangle \sigma^{\prime}(t) d t \\
= & \left\langle f(s)-y(s) \partial_{x} y(s), \phi\right\rangle \sigma(s)+\int_{s}^{T}\left\langle W(t-s)\left(f(s)-y(s) \partial_{x} y(s)\right), \mathcal{A}^{*} \phi\right\rangle \sigma(t) d t .
\end{aligned}
$$

Substituting (15)-16) into (13), we obtain

$$
-\int_{0}^{T}\langle y(t), \phi\rangle \sigma^{\prime}(t) d t=\int_{0}^{T}\left\langle y(t), \mathcal{A}^{*} \phi\right\rangle \sigma(t) d t+\int_{0}^{T}\left\langle f(t)-y(t) \partial_{x} y(s), \phi\right\rangle \sigma(t) d t .
$$

Due to Lemma 1.1, we have $y \partial_{x} y \in L^{1}\left(0, T ; L^{2}(0, L)\right)$. Furthermore, $y \in C\left([0, T] ; L^{2}(0, L)\right) \subset L^{1}\left(0, T ; L^{2}(0, L)\right)$, and $f \in L^{2}\left(0, T ; L^{2}(0, L)\right)$. Therefore $\langle y(\cdot), \phi\rangle \in W^{1,1}(0, T ; \mathbb{R})$ and for almost every $t \in[0, T]$ we have by (17)

$$
\frac{d}{d t}\langle y(t), \phi\rangle=\left\langle y(t), \mathcal{A}^{*} \phi\right\rangle-\left\langle y(t) \partial_{x} y(t), \phi\right\rangle+\langle f(t), \phi\rangle \quad \text { for all } \phi \in D\left(\mathcal{A}^{*}\right) .
$$

By Lemma 1.2 , we recall that $y \in \mathcal{W}_{0, T}$. Hence, we can rewrite (18) as

$$
\frac{d}{d t}\langle y(t), \phi\rangle_{H^{-2}, H^{2}}+\left\langle\partial_{x} y(t), \phi\right\rangle_{L^{2}}+\left\langle y(t) \partial_{x} y(t), \phi\right\rangle_{L^{2}}+\left\langle\partial_{x} y(t), \partial_{x}^{2} \phi\right\rangle_{L^{2}}=\langle f(t), \phi\rangle_{L^{2}} .
$$

Since $\mathcal{D}\left(\mathcal{A}^{*}\right)$ is dense in $\mathcal{X}$, the above equality holds for every $\phi \in \mathcal{X}$, and hence $y$ is a weak solution.

Now we show that every weak solution $(12)$ is a mild solution of $(4)$. By using the fact that $\mathcal{D}\left(\mathcal{A}^{*}\right) \subset \mathcal{X}$ and integrating by parts in 12 , we have for almost every $t \in[0, T]$

$$
\frac{d}{d t}\langle y(t), \phi\rangle=\left\langle y(t), \mathcal{A}^{*} \phi\right\rangle-\left\langle y(t) \partial_{x} y(t), \phi\right\rangle+\langle f(t), \phi\rangle \quad \text { for all } \phi \in D\left(\mathcal{A}^{*}\right) .
$$

Integrating on $(0, t)$ for an arbitrary $t \in[0, T]$, we obtain

$$
\left\langle y(t)-y_{0}+\int_{0}^{t}\left(y(s) \partial_{x} y(s)-f(s)\right) d s, \phi\right\rangle=\left\langle\int_{0}^{t} y(s) d s, \mathcal{A}^{*} \phi\right\rangle \quad \text { for all } \phi \in D\left(\mathcal{A}^{*}\right) .
$$

This equality implies that

$$
\left(\int_{0}^{t} y(s) d s, y(t)-y_{0}+\int_{0}^{t}\left(y(s) \partial_{x} y(s)-f(s)\right) d s\right) \in\left(\mathcal{I}\left(\mathcal{G}\left(A^{*}\right)\right)^{\perp} \quad \text { for all } t \in[0, T] .\right.
$$

By Lemma 1.3 , we can conclude that

$$
\left(\mathcal{I}\left(\mathcal{G}\left(A^{*}\right)\right)^{\perp}=\left((\mathcal{G}(A))^{\perp}\right)^{\perp}=\overline{\mathcal{G}(A)}=\mathcal{G}(A) .\right.
$$

Therefore, for all $t \in[0, T]$ we have $\int_{0}^{t} y(s) d s \in \mathcal{D}(\mathcal{A})$, and

$$
\mathcal{A} \int_{0}^{t} y(s) d s=y(t)-y_{0}+\int_{0}^{t}\left(y(s) \partial_{x} y(s)-f(s)\right) d s .
$$

Now by defining $z(t):=\int_{0}^{t} y(s) d s$ for all $t \in[0, T]$, we have

$$
\left\{\begin{array}{l}
\dot{z}(t)=\mathcal{A} z(t)+y_{0}-\int_{0}^{t}\left(y(s) \partial_{x} y(s)-f(s)\right) d s, \\
z(0)=0 .
\end{array}\right.
$$


We set $\mathcal{A}_{\lambda}=\lambda \mathcal{A}(\lambda I-\mathcal{A})^{-1}$ for $\lambda \in \mathbb{R}$ with $\lambda>0$ as the Yosida approximations of the operator $\mathcal{A}$. Then by 19 we can write

$$
\begin{aligned}
\frac{d}{d t}\left(e^{-\mathcal{A}_{\lambda} t} z(t)\right) & =e^{-\mathcal{A}_{\lambda} t} \dot{z}(t)-\mathcal{A}_{\lambda} e^{-\mathcal{A}_{\lambda} t} z(t) \\
& =\left(\mathcal{A}-\mathcal{A}_{\lambda}\right) e^{-\mathcal{A}_{\lambda} t} z(t)+e^{-\mathcal{A}_{\lambda} t}\left[y_{0}-\int_{0}^{t}\left(y(s) \partial_{x} y(s)-f(s)\right) d s\right] .
\end{aligned}
$$

It follows that

$$
\begin{aligned}
z(t) & =e^{\mathcal{A}_{\lambda} t} \int_{0}^{t}\left(\left(\mathcal{A}-\mathcal{A}_{\lambda}\right) e^{-\mathcal{A}_{\lambda} s} z(s)+e^{-\mathcal{A}_{\lambda} s}\left[y_{0}-\int_{0}^{s}\left(y(r) \partial_{x} y(r)-f(r)\right) d r\right]\right) d s \\
& =\int_{0}^{t}\left(\mathcal{A}-\mathcal{A}_{\lambda}\right) e^{\mathcal{A}_{\lambda}(t-s)} z(s) d s+\int_{0}^{t} e^{\mathcal{A}_{\lambda}(t-s)}\left[y_{0}-\int_{0}^{s}\left(y(r) \partial_{x} y(r)-f(r)\right) d r\right] d s .
\end{aligned}
$$

For every $\lambda>0$ and $s \in[0, T]$, by using 190 and Lemma 1.1 we have that

$$
\begin{aligned}
& \|\mathcal{A} z(s)\|_{L^{2}(0, L)} \leq\|y(s)\|_{L^{2}(0, L)}+\left\|y_{0}\right\|_{L^{2}(0, L)}+T^{\frac{1}{2}}\|f\|_{L^{2}\left(0, T ; L^{2}(0, L)\right)}+\left\|y \partial_{x} y\right\|_{L^{1}\left(0, T ; L^{2}(0, L)\right.} \leq C, \\
& \left\|\mathcal{A}_{\lambda} z(s)\right\|_{L^{2}(0, L)} \leq\left\|\lambda(\lambda I-\mathcal{A})^{-1}\right\|_{\mathcal{L}\left(L^{2}(0, L)\right)}\|\mathcal{A} z(s)\|_{L^{2}(0, L)} \leq\|\mathcal{A} z(s)\|_{L^{2}(0, L)} \leq C
\end{aligned}
$$

where, using that $y \in \mathcal{W}_{0, T}$, the constant $C$ is independent of $s \in[0, T]$. In addition,

$$
\begin{cases}\lim _{\lambda \rightarrow \infty}\left(\mathcal{A}-\mathcal{A}_{\lambda}\right) z(s)=0 & \text { for all } s \in[0, T] \\ \lim _{\lambda \rightarrow \infty} e^{\mathcal{A}_{\lambda} t} y=W(t) y & \text { for all } y \in L^{2}(0, L), \quad t \in[0, T] .\end{cases}
$$

Now by using the dominated convergence theorem and (21), we obtain from 20 for $\lambda \rightarrow \infty$

$$
\begin{aligned}
z(t) & =\int_{0}^{t} W(t-s)\left[y_{0}+\int_{0}^{s}\left(-y(r) \partial_{x} y(r)+f(r)\right) d r\right] d s \\
& =\int_{0}^{t} W(s) y_{0} d s+\int_{0}^{t} W(s)\left[\int_{0}^{t-s}\left(-y(r) \partial_{x} y(r)+f(r)\right) d r\right] d s .
\end{aligned}
$$

Therefore,

$$
y(t)=\dot{z}(t)=W(t) y_{0}+\int_{0}^{t} W(t-s)\left(-y(s) \partial_{x} y(s)+f(s)\right) d s,
$$

and thus $y$ is a mild solution. Finally uniqueness of the weak solution follows from the uniqueness of the mild solution.

\section{Existence of AN OPtimal CONTROL}

In Step 2 of any iteration of Algorithm 1, we need to solve a finite horizon optimal control problem consisting in minimizing

$$
J_{T}\left(u, y_{0}\right):=\int_{0}^{T} \ell(y(t), u(t)) d t,
$$

over all $u \in L^{2}\left(0, T ; L^{2}(\hat{\Omega})\right)$ subject to the nonlinear $\mathrm{KdV}$ equation

$$
\begin{cases}\partial_{t} y+\partial_{x} y+y \partial_{x} y+\partial_{x}^{3} y=B u & x \in(0, L), \quad t \in(0, T) \\ y(t, 0)=y(t, L)=\partial_{x} y(t, L)=0 & t \in(0, T) \\ y(0, \cdot)=y_{0} & x \in(0, L)\end{cases}
$$


where $y_{0} \in L^{2}(0, L)$. Therefore we need to verify that the above optimal control problem has a solution. This question will be addressed by the following theorem. We denote the above optimal control problem by $(\mathrm{OP})$ and write it as

$$
\min \left\{J_{T}\left(u, y_{0}\right) \mid(y, u) \text { satisfies } 22, u \in L^{2}\left(0, T ; L^{2}(\hat{\Omega})\right)\right\}
$$

Theorem 2.1. For every finite horizon $T>0$ and $y_{0} \in L^{2}(0, L)$, the optimal control problem (OP) admits a solution.

Proof. According to Theorem 1.4 for every control $u \in L^{2}\left(0, T ; L^{2}(\hat{\Omega})\right)$ there exist a unique weak solution $y \in \mathcal{W}_{0, T}$ to 22 . As a result, the set of admissible controls is nonempty and by (3) we have

$$
J_{T}\left(u, y_{0}\right) \geq \frac{\beta}{2}\|u\|_{L^{2}\left(0, T ; L^{2}(\hat{\Omega})\right)}^{2}
$$

Let $\left(y^{n}, u^{n}\right) \in \mathcal{W}_{0, T} \times L^{2}\left(0, T ; L^{2}(\hat{\Omega})\right)$ be a minimizing sequence such that

$$
\lim _{n \rightarrow \infty} J_{T}\left(u^{n}, y_{0}\right)=\sigma
$$

By (11), (23), and due to the structure of $\ell$, the set $\left\{\left(y^{n}, u^{n}\right)\right\}_{n}$ is bounded in $\mathcal{W}_{0, T} \times L^{2}\left(0, T ; L^{2}(\hat{\Omega})\right)$. Therefore there exist subsequences $y^{n}$ and $u^{n}$ such that

$$
\begin{aligned}
& y^{n} \rightarrow^{*} y^{*} \text { in } L^{2}\left(0, T ; H_{0}^{1}(0, L)\right) \cap L^{\infty}\left(0, T ; L^{2}(0, L)\right) \cap H^{1}\left(0, T ; H^{-2}(0, L)\right), \\
& u^{n} \rightarrow u^{*} \text { in } L^{2}\left(0, T ; L^{2}(\hat{\Omega})\right),
\end{aligned}
$$

where

$$
\begin{aligned}
& y^{*} \in L^{2}\left(0, T ; H_{0}^{1}(0, L)\right) \cap L^{\infty}\left(0, T ; L^{2}(0, L)\right) \cap H^{1}\left(0, T ; H^{-2}(0, L)\right), \\
& u^{*} \in L^{2}\left(0, T ; L^{2}(\hat{\Omega})\right) .
\end{aligned}
$$

It remains to show that $y^{*}$ is the weak solution to 22 corresponding to control $u^{*}$. By definition of weak convergence we have

$$
\int_{0}^{T}\left\langle\partial_{t} y^{n}-\partial_{t} y^{*}, \phi\right\rangle_{H^{-2}, H^{2}} d t \rightarrow 0 \quad \text { for every } \phi \in L^{2}(0, T ; \mathcal{X})
$$

By the compact embedding [54 of $L^{2}\left(0, T ; H_{0}^{1}(0, L)\right) \cap H^{1}\left(0, T ; H^{-2}(0, L)\right)$ into $L^{2}\left(0, T ; L^{2}(0, L)\right)$, we obtain for every $\phi \in L^{2}(0, T ; \mathcal{X})$

$$
\begin{aligned}
& \int_{0}^{T} \int_{0}^{L}\left(y^{n} \partial_{x} y^{n}-y^{*} \partial_{x} y^{*}\right) \phi d x d t=-\frac{1}{2} \int_{0}^{T} \int_{0}^{L}\left(\left(y^{n}\right)^{2}-\left(y^{*}\right)^{2}\right) \partial_{x} \phi d x d t \\
& \quad \leq \frac{1}{2} \int_{0}^{T}\left(\left\|y^{n}(t)\right\|_{L^{2}(0, L)}+\left\|y^{*}(t)\right\|_{L^{2}(0, L)}\right)\left\|y^{n}(t)-y^{*}(t)\right\|_{L^{2}(0, L)}\left\|\partial_{x} \phi(t)\right\|_{L^{\infty}(0, L)} d t \\
& \quad \leq \frac{1}{2} c_{5}\left(\left\|y^{n}\right\|_{C\left([0, T] ; L^{2}(0, L)\right)}+\left\|y^{*}\right\|_{C\left([0, T] ; L^{2}(0, L)\right)}\right)\left\|y^{n}-y^{*}\right\|_{L^{2}\left(0, T ; L^{2}(0, L)\right)}\|\phi\|_{L^{2}\left(0, T, H^{2}(0, T)\right)} \rightarrow 0
\end{aligned}
$$

where the constant $c_{5}$ stands for the continuous embedding of $H^{2}(0, L)$ into $W^{1, \infty}(0, L)$. By (24) we obtain

$$
\int_{0}^{T}\left\langle B u^{n}-B u^{*}, \phi\right\rangle d t \rightarrow 0 \quad \text { for all } \phi \in L^{2}(0, T ; \mathcal{X}) .
$$

Due to the fact that $y^{*}(0) \in L^{2}(0, L)$ and using (25), (26), and (12) with $f=B u$, we conclude that $y^{*} \in \mathcal{W}_{0, T}$ is the weak solution to 22 corresponding to $u^{*}$. Since $y^{n} \rightarrow y^{*}$ strongly in $L^{2}\left(0, T ; L^{2}(0, L)\right)$ 
and $u^{n} \rightarrow u^{*}$ weakly in $L^{2}\left(0, T ; L^{2}(\hat{\Omega})\right)$ we have

$$
0 \leq J_{T}\left(u^{*}, y_{0}\right) \leq \liminf _{n \rightarrow \infty} J_{T}\left(u^{n}, y_{0}\right)=\sigma,
$$

and as a consequence the pair $\left(y^{*}, u^{*}\right)$ is optimal.

\section{Semi-Global stabilizability of KDV}

In this section, we review some results about the stablizability of the nonlinear KdV equation by feedback. We consider

$$
\begin{cases}\partial_{t} y+\partial_{x} y+\partial_{x}^{3} y+y \partial_{x} y=F(y) & x \in(0, L), \quad t \in(0, T) \\ y(t, 0)=y(t, L)=\partial_{x} y(t, L)=0 & t \in(0, T) \\ y(0, \cdot)=y_{0} & x \in(0, L)\end{cases}
$$

where $F$ is linear feedback control which acts only on a subdomain of $[0, L]$. Our objective is to find a control which dissipates enough energy to force the decay of the solution with respect to the $L^{2}$-norm. The control is of the form $F(y)=-\omega y$, where $\omega$ is defined by

$$
\left\{\begin{array}{c}
\omega \in L^{\infty}(0, L) \text { and } \omega(x) \geq \omega_{0}>0 \text { for a.e. in } \hat{\Omega} \\
\text { where } \hat{\Omega} \text { is any nonempty open subset of }[0, L] .
\end{array}\right.
$$

In [49 Rosier studied the controllability of the linear KdV equation and he found the set of critical points which is given by

$$
\Upsilon:=\left\{2 \pi \sqrt{\frac{k^{2}+k l+l^{2}}{3}} \mid k, l \in \mathbb{N}\right\} .
$$

Moreover, he discovered that, if the length $L$ of the spatial domain belongs to set $\Upsilon$, the uncontrolled $(\omega=0)$ linear $\mathrm{KdV}$ equation has solutions for which the $L^{2}$-norm stays constant as $t \rightarrow \infty$. In this case, i.e., $L \in \Upsilon$, one can show that the linear $\mathrm{KdV}$ equation is globally exponentially stabilizable by a linear feedback law of the form $F(y)=-\omega y$ acting on an open subset $\hat{\Omega}$ of $[0, L]$, see, e.g., 45$]$.

For the nonlinear KdV equation, the situation is more delicate and it is not clear whether the solutions goes to zero. In [45] by using a perturbation argument it has been shown that the nonlinear KdV is locally stabilizable for small initial functions. Alternative approaches [45,46] are directly dealing with the semiglobal stabilizability of the nonlinear KdV equation.

Theorem 3.1 (see [46]). Let $L>0$ and $\omega=\omega(x)$ be defined by (28). Then by setting $F(y)=-\omega y$ as a feedback control in (27), the resulting closed loop system is semi-globally exponentially stable. That is, for every $r>0$ there exist $c=c(r)$ and $\mu=\mu(r)$ such that

$$
\|y(t)\|_{L^{2}(0, L)}^{2} \leq c\left\|y_{0}\right\|_{L^{2}(0, L)}^{2} e^{-\mu t}
$$

holds for all $t>0$ and any initial function $y_{0} \in L^{2}(0, L)$ with $\left\|y_{0}\right\|_{L^{2}(0, L)} \leq r$.

The following estimates will be used later.

Lemma 3.1. Consider the controlled system (2). Then for every control $u \in L^{2}\left(0, T ; L^{2}(\hat{\Omega})\right)$ and $y_{0} \in$ $L^{2}(0, L)$ we have the following estimate

$$
\|y(t)\|_{L^{2}(0, L)}^{2} \leq\left\|y_{0}\right\|_{L^{2}(0, L)}^{2}+\int_{0}^{t}\|y(s)\|_{L^{2}(0, L)}^{2} d s+\int_{0}^{t}\|u(s)\|_{L^{2}(\hat{\Omega})}^{2} d s \quad \text { for all } t \in[0, T] .
$$

Moreover for every $\delta \in(0, T]$ we have

$$
\|y(\delta)\|_{L^{2}(0, L)}^{2} \leq c_{\delta} \int_{0}^{\delta}\left(\|y(s)\|_{L^{2}(0, L)}^{2}+\|u(s)\|_{L^{2}(\hat{\Omega})}^{2}\right) d s,
$$


where the constant $c_{\delta}$ depends only on $\delta$.

Proof. Assume that $q \in C^{\infty}([0, T] \times[0, L])$ and that the solution $y$ of $(2)$ is regular enough to justify the following computations. Multiplying both sides of the equation by $y q$ and integrating over $(0, t) \times(0, L)$ for an arbitrary $t \in[0, T]$ we obtain

$$
\int_{0}^{t} \int_{0}^{L} q y\left(\partial_{t} y+\partial_{x} y+\partial_{x}^{3} y+y \partial_{x} y-B u\right) d x d s=0
$$

Integration by parts and use of the boundary conditions implies that

$$
\begin{aligned}
& -\int_{0}^{t} \int_{0}^{L}\left(\partial_{t} q+\partial_{x} q+\partial_{x}^{3} q\right) \frac{y^{2}}{2} d x d s-\frac{1}{3} \int_{0}^{t} \int_{0}^{L} y^{3} \partial_{x} q d x d s+\int_{0}^{L}\left(q \frac{y^{2}}{2}\right)(t, x) d x \\
& -\int_{0}^{L} q(0, x) \frac{y_{0}^{2}(x)}{2} d x+\frac{3}{2} \int_{0}^{t} \int_{0}^{L} \partial_{x} q\left(\partial_{x} y\right)^{2} d x d s+\int_{0}^{t}\left(q \frac{\left(\partial_{x} y\right)^{2}}{2}\right)(s, 0) d s \\
& -\int_{0}^{t} \int_{0}^{L} y q B u d x d s=0 .
\end{aligned}
$$

For the choice $q:=1$, we obtain

$$
\begin{aligned}
\|y(t)\|_{L^{2}(0, L)}^{2}+\int_{0}^{t}\left(\partial_{x} y\right)^{2}(s, 0) d s & =\left\|y_{0}\right\|_{L^{2}(0, L)}^{2}+2 \int_{0}^{t}\langle y(s), u(s)\rangle_{L^{2}(0, L)} d s \\
& \leq\left\|y_{0}\right\|_{L^{2}(0, L)}^{2}+2 \int_{0}^{t}\|y(t)\|_{L^{2}(0, L)}\|u(t)\|_{L^{2}(\hat{\Omega})} d s \\
& \leq\left\|y_{0}\right\|_{L^{2}(0, L)}^{2}+\int_{0}^{t}\|y(s)\|_{L^{2}(0, L)}^{2} d s+\int_{0}^{t}\|u(s)\|_{L^{2}(\hat{\Omega})}^{2} d s
\end{aligned}
$$

By a density argument we obtain (29).

Turning to inequality (30), by choosing $t=\delta, q:=\delta-s$ with $s \in(0, \delta)$ for a fixed $\delta \in(0, T]$ in 31 we obtain

$$
\begin{aligned}
\frac{1}{2} \delta\left\|y_{0}\right\|_{L^{2}(0, L)}^{2} & =\frac{1}{2} \int_{0}^{\delta}\|y(s)\|_{L^{2}(0, L)}^{2} d s+\frac{1}{2} \int_{0}^{\delta}(\delta-s)\left(\partial_{x} y\right)^{2}(s, 0) d s-\int_{0}^{\delta} \int_{0}^{L}(\delta-s) y B u d x d s \\
& \leq \frac{1}{2} \int_{0}^{\delta}\|y(s)\|_{L^{2}(0, L)}^{2} d s+\frac{\delta}{2} \int_{0}^{\delta}\left(\partial_{x} y\right)^{2}(s, 0) d s+\delta \int_{0}^{\delta}\left|\langle y(s), B u(s)\rangle_{L^{2}}\right| d s \\
& \leq \frac{1}{2} \int_{0}^{\delta}\|y(s)\|_{L^{2}(0, L)}^{2} d s+\frac{\delta}{2} \int_{0}^{\delta}\left(\partial_{x} y\right)^{2}(s, 0) d s+\frac{\delta}{2} \int_{0}^{\delta}\left(\|y(s)\|_{L^{2}(0, L)}^{2}+\|u(s)\|_{L^{2}(\hat{\Omega})}^{2}\right) d s \\
& \leq \frac{\delta}{2} \int_{0}^{\delta}\left(\partial_{x} y\right)^{2}(s, 0) d s+\frac{\delta+1}{2} \int_{0}^{\delta}\left(\|y(s)\|_{L^{2}(0, L)}^{2}+\|u(s)\|_{L^{2}(\hat{\Omega})}^{2}\right) d s
\end{aligned}
$$

and as consequence we can write

$$
-\int_{0}^{\delta}\left(\partial_{x} y(s, 0)\right)^{2} d s \leq-\left\|y_{0}\right\|_{L^{2}(0, L)}^{2}+\frac{\delta+1}{\delta} \int_{0}^{\delta}\left(\|y(s)\|_{L^{2}(0, L)}^{2}+\|u(s)\|_{L^{2}(\hat{\Omega})}^{2}\right) d s .
$$

Moreover, by using 32 for $t=\delta$ we infer that

$$
\|y(\delta)\|_{L^{2}(0, L)}^{2} \leq\left\|y_{0}\right\|_{L^{2}(0, L)}^{2}-\int_{0}^{\delta}\left(\partial_{x} y(s, 0)\right)^{2} d s+\int_{0}^{\delta}\left(\|y(s)\|_{L^{2}(0, L)}^{2} d s+\|u(s)\|_{L^{2}(\hat{\Omega})}^{2}\right) d s
$$


By combining (33) and (34), we have

$$
\|y(\delta)\|_{L^{2}(0, L)}^{2} \leq \frac{2 \delta+1}{\delta} \int_{0}^{\delta}\left(\|y(s)\|_{L^{2}(0, L)}^{2}+\|u(s)\|_{L^{2}(\hat{\Omega})}^{2}\right) d s
$$

and with $c_{\delta}:=\frac{2 \delta+1}{\delta}$, we conclude the proof.

Definition 3.1. For any $y_{0} \in L^{2}(0, L)$ the infinite horizon value function $V_{\infty}(\cdot)$ is defined as the extended real valued function

$$
\left.V_{\infty}\left(y_{0}\right):=\inf _{u \in L^{2}\left(0, \infty ; L^{2}(\hat{\Omega})\right)}\left\{J_{\infty}\left(u, y_{0}\right) \text { subject to } 2\right)\right\} .
$$

Similarly, the finite horizon value function $V_{T}(\cdot)$ is defined by

$$
V_{T}\left(y_{0}\right):=\min _{u \in L^{2}\left(0, T ; L^{2}(\hat{\Omega})\right)}\left\{J_{T}\left(u, y_{0}\right) \text { subject to }(2)\right\} \text {. }
$$

From this point forward, $\mathcal{B}_{r}(0)$ denotes a ball in $L^{2}(0, L)$ centered at 0 with radius $r$ and we define $\alpha_{\ell}:=\frac{\min \{\beta, 1\}}{2}$. Furthermore, the pair $\left(y_{T}^{*}\left(\cdot ; y_{0}, t_{0}\right), u_{T}^{*}\left(\cdot ; y_{0}, t_{0}\right)\right)$ stands for an optimal solution to the problem $\mathrm{OP}$ with finite time horizon $T$, and initial function $y_{0}$ at initial time $t_{0}$. In the following the function

$$
\gamma(T, r):=\frac{(1+\beta) c(r)}{2 \mu(r)}\left(1-e^{-\mu(r) T}\right)
$$

with $c(r)$ and $\mu(r)$ from Theorem 3.1 will be of significance. For every $r>0$, it is nondecreasing, continuous, and bounded function in the variable $T$.

Lemma 3.2. Let a positive number $r$ be given. Then for every $y_{0} \in \mathcal{B}_{r}(0) \subset L^{2}(0, L)$ and $T>0$, there exists a control $\hat{u}\left(\cdot ; y_{0}\right) \in L^{2}\left(0, T ; L^{2}(\hat{\Omega})\right)$ such that

$$
V_{T}\left(y_{0}\right) \leq J_{T}\left(\hat{u}, y_{0}\right) \leq \gamma(T, r)\left\|y_{0}\right\|_{L^{2}(0, L)}^{2} .
$$

Proof. Assume that positive numbers $r, T$, and $y_{0} \in \mathcal{B}_{r}(0)$ are given. By setting $u(t):=-\left.y(t)\right|_{\hat{\Omega}}$ in the controlled system (2), and using Theorem 3.1 for the choice

$$
\omega(x):= \begin{cases}1 & x \in \hat{\Omega} \\ 0 & \text { otherwise }\end{cases}
$$

we obtain

$$
\|y(t)\|_{L^{2}(0, L)}^{2} \leq c(r)\left\|y_{0}\right\|_{L^{2}(0, L)}^{2} e^{-\mu(r) t} \quad \text { for all } t \in[0, T] .
$$

Here the constants $c(r)$ and $\mu(r)$ were defined in Theorem 3.1. By integrating from 0 to $T$ we have

$$
\int_{0}^{T}\|y(t)\|_{L^{2}(0, L)}^{2} d t \leq \frac{c(r)}{\mu(r)}\left(1-e^{-\mu(r) T}\right)\left\|y_{0}\right\|_{L^{2}(0, L)}^{2} .
$$

By the definition of value function $V_{T}(\cdot)$ and $(3)$ we have

$$
\begin{aligned}
V_{T}\left(y_{0}\right) \leq \int_{0}^{T} \frac{1}{2}\|y(t)\|_{L^{2}(0, L)}^{2}+\frac{\beta}{2}\|y(t)\|_{L^{2}(\hat{\Omega})}^{2} d t & \leq \frac{(1+\beta) c(r)}{2 \mu(r)}\left(1-e^{-\mu(r) T}\right)\left\|y_{0}\right\|_{L^{2}(0, L)}^{2} \\
& =\gamma(T, r)\left\|y_{0}\right\|_{L^{2}(0, L)}^{2} .
\end{aligned}
$$


Lemma 3.3. Let $r_{0}>0, \delta>0$, and $T>\delta$ be given. Then there exists a radius $d_{1}$ depending on $r_{0}$ such that for every $r \geq d_{1}\left(r_{0}\right)$ and $y_{0} \in \mathcal{B}_{r_{0}}(0)$ the following inequities are satisfied

$$
\begin{aligned}
V_{T}\left(y_{T}^{*}\left(\delta ; y_{0}, 0\right)\right) \leq \int_{\delta}^{\tilde{t}} \ell\left(y_{T}^{*}\left(t ; y_{0}, 0\right), u_{T}^{*}\left(t ; y_{0}, 0\right)\right) d t \\
\\
\quad+\gamma(T+\delta-\tilde{t}, r)\left\|y_{T}^{*}\left(\tilde{t} ; y_{0}, 0\right)\right\|_{L^{2}(0, L)}^{2} \quad \text { for all } \tilde{t} \in[\delta, T],
\end{aligned}
$$

and

$$
\int_{\tilde{t}}^{T} \ell\left(y_{T}^{*}\left(t ; y_{0}, 0\right), u_{T}^{*}\left(t ; y_{0}, 0\right)\right) d t \leq \gamma(T-\tilde{t}, r)\left\|y_{T}^{*}\left(\tilde{t} ; y_{0}, 0\right)\right\|_{L^{2}(0, L)}^{2} \quad \text { for all } \tilde{t} \in[0, T] .
$$

Proof. For every $y_{0} \in L^{2}(0, L)$ and $\tilde{t} \in[0, T]$, due to $(3)$ and Bellman's optimality principle we have

$$
\begin{aligned}
\alpha_{\ell} \int_{0}^{\tilde{t}}\left(\left\|y_{T}^{*}\left(t ; y_{0}, 0\right)\right\|_{L^{2}(0, L)}^{2}+\left\|u_{T}^{*}\left(t ; y_{0}, 0\right)\right\|_{L^{2}(\hat{\Omega})}^{2}\right) d t & \leq \int_{0}^{\tilde{t}} \ell\left(y_{T}^{*}\left(t ; y_{0}, 0\right), u_{T}^{*}\left(t ; y_{0}, 0\right)\right) d t \\
& =V_{T}\left(y_{0}\right)-V_{T-\tilde{t}}\left(y_{T}^{*}\left(\tilde{t} ; y_{0}, 0\right)\right) .
\end{aligned}
$$

Now by 29, 35 and the above inequality we have for $y_{0} \in \mathcal{B}_{r_{0}}(0)$

$$
\begin{aligned}
\left\|y_{T}^{*}\left(\tilde{t} ; y_{0}, 0\right)\right\|_{L^{2}(0, L)}^{2} & \leq\left\|y_{0}\right\|_{L^{2}(0, L)}^{2}+\int_{0}^{\tilde{t}}\left\|y_{T}^{*}\left(t ; y_{0}, 0\right)\right\|_{L^{2}(0, L)}^{2} d t+\int_{0}^{\tilde{t}}\left\|u_{T}^{*}\left(t ; y_{0}, 0\right)\right\|_{L^{2}(\hat{\Omega})}^{2} d t \\
& \leq\left\|y_{0}\right\|_{L^{2}(0, L)}^{2}+\frac{1}{\alpha_{\ell}}\left(V_{T}\left(y_{0}\right)-V_{T-\tilde{t}}\left(y_{T}^{*}\left(\tilde{t} ; y_{0}, 0\right)\right)\right. \\
& \leq\left\|y_{0}\right\|_{L^{2}(0, L)}^{2}+\frac{1}{\alpha_{\ell}} V_{T}\left(y_{0}\right) \leq\left(1+\frac{\gamma\left(T, r_{0}\right)}{\alpha_{\ell}}\right) r_{0}^{2} \\
& \leq\left(1+\frac{(1+\beta) c\left(r_{0}\right)}{2 \alpha_{\ell} \mu\left(r_{0}\right)}\right) r_{0}^{2}=: d_{1}^{2}\left(r_{0}\right)
\end{aligned}
$$

Hence for the radius $d_{1}$ defined in the above inequality, we have

$$
y_{T}^{*}\left(\tilde{t} ; y_{0}, 0\right) \in \mathcal{B}_{d_{1}}(0) \quad \text { for all } \tilde{t} \in[0, T]
$$

We turn to the verification of (37). For simplicity of notation, we denote $y_{T}^{*}\left(\delta ; y_{0}, 0\right)$ by $y^{*}(\delta)$. Then for every fixed $r \geq d_{1}$ we have $y_{0} \in \mathcal{B}_{r}(0)$. Due to Bellman's optimality principle, we have for every $\tilde{t} \in[\delta, T]$

$$
\begin{aligned}
V_{T} & \left(y^{*}(\delta)\right)=\int_{\delta}^{T+\delta} \ell\left(y_{T}^{*}\left(t ; y^{*}(\delta), \delta\right), u_{T}^{*}\left(t ; y^{*}(\delta), \delta\right)\right) d t \\
\quad & =\int_{\delta}^{\tilde{t}} \ell\left(y_{T}^{*}\left(t ; y^{*}(\delta), \delta\right), u_{T}^{*}\left(t ; y^{*}(\delta), \delta\right)\right) d t+V_{T+\delta-\tilde{t}}\left(y_{T}^{*}\left(\tilde{t} ; y^{*}(\delta), \delta\right)\right) .
\end{aligned}
$$

By optimality of $y_{T}^{*}\left(\cdot ; y^{*}(\delta), \delta\right)$ as a solution on $[\delta, T+\delta]$ with initial state $y^{*}(\delta) \in \mathcal{B}_{d_{1}}(0) \subseteq \mathcal{B}_{r}(0)$ at $t=\delta$ we obtain

$$
\begin{aligned}
V_{T}\left(y^{*}(\delta)\right) & \leq \int_{\delta}^{\tilde{t}} \ell\left(y_{T}^{*}\left(t ; y_{0}, 0\right), u_{T}^{*}\left(t ; y_{0}, 0\right)\right) d t+V_{T+\delta-\tilde{t}}\left(y_{T}^{*}\left(\tilde{t} ; y_{0}, 0\right)\right) \\
& \leq \int_{\delta}^{\tilde{t}} \ell\left(y_{T}^{*}\left(t ; y_{0}, 0\right), u_{T}^{*}\left(t ; y_{0}, 0\right)\right) d t+\gamma(T+\delta-\tilde{t}, r)\left\|y_{T}^{*}\left(\tilde{t} ; y_{0}, 0\right)\right\|_{L^{2}(0, L)}^{2},
\end{aligned}
$$

where for the last inequality we used 35 . 
To prove the second inequality, let $\tilde{t} \in[0, T]$ be arbitrary. By Bellman's principle and (35), we have

$$
\begin{aligned}
V_{T}\left(y_{0}\right) & =\int_{0}^{\tilde{t}} \ell\left(y_{T}^{*}\left(t ; y_{0}, 0\right), u_{T}^{*}\left(t ; y_{0}, 0\right)\right) d t+\int_{\tilde{t}}^{T} \ell\left(y_{T}^{*}\left(t ; y_{0}, 0\right), u_{T}^{*}\left(t ; y_{0}, 0\right)\right) d t \\
& =\int_{0}^{\tilde{t}} \ell\left(y_{T}^{*}\left(t ; y_{0}, 0\right), u_{T}^{*}\left(t ; y_{0}, 0\right)\right) d t+V_{T-\tilde{t}}\left(y_{T}^{*}\left(\tilde{t} ; y_{0}, 0\right)\right) \\
& \leq \int_{0}^{\tilde{t}} \ell\left(y_{T}^{*}\left(t ; y_{0}, 0\right), u_{T}^{*}\left(t ; y_{0}, 0\right)\right) d t+\gamma(T-\tilde{t}, r)\left\|y_{T}^{*}\left(\tilde{t} ; y_{0}, 0\right)\right\|_{L^{2}(0, L)}^{2} .
\end{aligned}
$$

Therefore,

$$
\int_{\tilde{t}}^{T} \ell\left(y_{T}^{*}\left(t ; y_{0}, 0\right), u_{T}^{*}\left(t ; y_{0}, 0\right)\right) d t \leq \gamma(T-\tilde{t}, r)\left\|y_{T}^{*}\left(\tilde{t} ; y_{0}, 0\right)\right\|_{L^{2}(0, L)}^{2} \quad \text { for all } \tilde{t} \in[0, T],
$$

as desired.

Lemma 3.4. Suppose that $r_{0}>0, \delta>0$, and $T>\delta$ are given. Then for every $r \geq d_{1}\left(r_{0}\right)$ with $d_{1}$ defined in Lemma 3.3, and the choices

$$
\theta_{1}(\delta, T, r):=1+\frac{\gamma(T, r)}{\alpha_{\ell}(T-\delta)}, \quad \theta_{2}(\delta, T, r):=\frac{\gamma(T, r)}{\alpha_{\ell} \delta},
$$

the estimates

$$
V_{T}\left(y_{T}^{*}\left(\delta ; y_{0}, 0\right)\right) \leq \theta_{1} \int_{\delta}^{T} \ell\left(y_{T}^{*}\left(t ; y_{0}, 0\right), u_{T}^{*}\left(t ; y_{0}, 0\right)\right) d t
$$

and

$$
\int_{\delta}^{T} \ell\left(y_{T}^{*}\left(t ; y_{0}, 0\right), u_{T}^{*}\left(t ; y_{0}, 0\right)\right) d t \leq \theta_{2} \int_{0}^{\delta} \ell\left(y_{T}^{*}\left(t ; y_{0}, 0\right), u_{T}^{*}\left(t ; y_{0}, 0\right)\right) d t
$$

hold for every $y_{0} \in \mathcal{B}_{r_{0}}(0)$.

Proof. According to Lemma 3.3 the estimates 37 and 38 are satisfied for every $y_{0} \in \mathcal{B}_{r_{0}}(0)$ and $r \geq d_{1}\left(r_{0}\right)$.

We first verify inequality (40) for arbitrary initial function $y_{0} \in \mathcal{B}_{r_{0}}(0)$ and $r \geq d_{1}\left(r_{0}\right)$. Recall that for the solution of the $\mathrm{KdV}$ equation we have $y_{T}^{*}\left(\cdot ; y_{0}, 0\right) \in C\left([0, T] ; L^{2}(0, L)\right)$. Hence there is a $\bar{t} \in[\delta, T]$ such that

$$
\bar{t}=\arg \min _{t \in[\delta, T]}\left\|y_{T}^{*}\left(t ; y_{0}, 0\right)\right\|_{L^{2}(0, L)}^{2}
$$

By (37) we have

$$
\begin{aligned}
V_{T}\left(y_{T}^{*}\right. & \left.\left(\delta ; y_{0}, 0\right)\right) \\
& \leq \int_{\delta}^{\bar{t}} \ell\left(y_{T}^{*}\left(t ; y_{0}, 0\right), u_{T}^{*}\left(t ; y_{0}, 0\right)\right) d t+\gamma(T+\delta-\bar{t}, r)\left\|y_{T}^{*}\left(\bar{t} ; y_{0}, 0\right)\right\|_{L^{2}(0, L)}^{2} \\
& \leq \int_{\delta}^{T} \ell\left(y_{T}^{*}\left(t ; y_{0}, 0\right), u_{T}^{*}\left(t ; y_{0}, 0\right)\right) d t+\gamma(T, r)\left\|y_{T}^{*}\left(\bar{t} ; y_{0}, 0\right)\right\|_{L^{2}(0, L)}^{2} \\
& \leq \int_{\delta}^{T} \ell\left(y_{T}^{*}\left(t ; y_{0}, 0\right), u_{T}^{*}\left(t ; y_{0}, 0\right)\right) d t+\frac{\gamma(T, r)}{T-\delta} \int_{\delta}^{T}\left\|y_{T}^{*}\left(t ; y_{0}, 0\right)\right\|_{L^{2}(0, L)}^{2} d t .
\end{aligned}
$$

Furthermore, by (3)

$$
\int_{\delta}^{T}\left\|y_{T}^{*}\left(t ; y_{0}, 0\right)\right\|_{L^{2}(0, L)}^{2} d t \leq \frac{1}{\alpha_{\ell}} \int_{\delta}^{T} \ell\left(y_{T}^{*}\left(t ; y_{0}, 0\right), u_{T}^{*}\left(t ; y_{0}, 0\right)\right) d t .
$$


By 42 and 43 we have

$$
V_{T}\left(y_{T}^{*}\left(\delta ; y_{0}, 0\right)\right) \leq\left(1+\frac{\gamma(T, r)}{\alpha_{\ell}(T-\delta)}\right) \int_{\delta}^{T} \ell\left(y_{T}^{*}\left(t ; y_{0}, 0\right), u_{T}^{*}\left(t ; y_{0}, 0\right)\right) d t .
$$

Turning to 41) we define

$$
\hat{t}=\arg \min _{t \in[0, \delta]}\left\|y_{T}^{*}\left(t ; y_{0}, 0\right)\right\|_{L^{2}(0, L)}^{2}
$$

Then by (38) we have

$$
\begin{aligned}
\int_{\delta}^{T} \ell\left(y_{T}^{*}\left(t ; y_{0}, 0\right)\right. & \left., u_{T}^{*}\left(t ; y_{0}, 0\right)\right) d t \\
& \leq \int_{\hat{t}}^{T} \ell\left(y_{T}^{*}\left(t ; y_{0}, 0\right), u_{T}^{*}\left(t ; y_{0}, 0\right)\right) d t \leq \gamma(T-\hat{t}, r)\left\|y_{T}^{*}\left(\hat{t} ; y_{0}, 0\right)\right\|_{L^{2}(0, L)}^{2} \\
& \leq \gamma(T, r)\left\|y_{T}^{*}\left(\hat{t} ; y_{0}, 0\right)\right\|_{L^{2}(0, L)}^{2} \leq \frac{\gamma(T, r)}{\delta} \int_{0}^{\delta}\left\|y_{T}^{*}\left(t ; y_{0}, 0\right)\right\|_{L^{2}(0, L)}^{2} d t
\end{aligned}
$$

and further

$$
\frac{\gamma(T, r)}{\delta} \int_{0}^{\delta}\left\|y_{T}^{*}\left(t ; y_{0}, 0\right)\right\|_{L^{2}(0, L)}^{2} d t \leq \frac{\gamma(T, r)}{\alpha_{\ell} \delta} \int_{0}^{\delta} \ell\left(y_{T}^{*}\left(t ; y_{0}, 0\right), u_{T}^{*}\left(t ; y_{0}, 0\right)\right) d t .
$$

By (44) and 45] we obtain the desired estimate

$$
\int_{\delta}^{T} \ell\left(y_{T}^{*}\left(t ; y_{0}, 0\right), u_{T}^{*}\left(t ; y_{0}, 0\right)\right) d t \leq \frac{\gamma(T, r)}{\alpha_{\ell} \delta} \int_{0}^{\delta} \ell\left(y_{T}^{*}\left(t ; y_{0}, 0\right), u_{T}^{*}\left(t ; y_{0}, 0\right)\right) d t .
$$

Proposition 3.1. Suppose that $r_{0}>0$ and $\delta>0$ are given. Then for every $r \geq d_{1}\left(r_{0}\right)$ with $d_{1}$ defined in Lemma 3.3. there exist positive numbers $T^{*}=T^{*}(r, \delta)>\delta$, and $\alpha=\alpha(r, \delta) \in(0,1)$ such that

$$
V_{T}\left(y_{T}^{*}\left(\delta ; y_{0}, 0\right)\right) \leq V_{T}\left(y_{0}\right)-\alpha \int_{0}^{\delta} \ell\left(y_{T}^{*}\left(t ; y_{0}, 0\right), u_{T}^{*}\left(t ; y_{0}, 0\right)\right) d t
$$

for every $T \geq T^{*}$ and $y_{0} \in \mathcal{B}_{r_{0}}(0)$.

Proof. From the definition of $V_{T}\left(y_{0}\right)$ and Lemma 3.4 , we have for every $r \geq d_{1}$

$$
\begin{aligned}
& V_{T}\left(y_{T}^{*}\left(\delta ; y_{0}, 0\right)\right)-V_{T}\left(y_{0}\right)=V_{T}\left(y_{T}^{*}\left(\delta ; y_{0}, 0\right)\right)-\int_{0}^{T} \ell\left(y_{T}^{*}\left(t ; y_{0}, 0\right), u_{T}^{*}\left(t ; y_{0}, 0\right)\right) d t \\
& \quad \leq\left(\theta_{1}-1\right) \int_{\delta}^{T} \ell\left(y_{T}^{*}\left(t ; y_{0}, 0\right), u_{T}^{*}\left(t ; y_{0}, 0\right)\right) d t-\int_{0}^{\delta} \ell\left(y_{T}^{*}\left(t ; y_{0}, 0\right), u_{T}^{*}\left(t ; y_{0}, 0\right)\right) d t \\
& \quad \leq\left(\theta_{2}\left(\theta_{1}-1\right)-1\right) \int_{0}^{\delta} \ell\left(y_{T}^{*}\left(t ; y_{0}, 0\right), u_{T}^{*}\left(t ; y_{0}, 0\right)\right) d t
\end{aligned}
$$

where $\theta_{1}$ and $\theta_{2}$ are defined in Lemma 3.4. Since

$$
1-\theta_{2}\left(\theta_{1}-1\right)=1-\frac{\gamma^{2}(T, r)}{\alpha_{\ell}^{2} \delta(T-\delta)}
$$

and

$$
\frac{\gamma^{2}(T, r)}{\alpha_{\ell}^{2} \delta(T-\delta)} \rightarrow 0 \text { as } T \rightarrow \infty
$$

there exist $T^{*}>\delta$ and $\alpha \in(0,1)$ such that $1-\theta_{2}\left(\theta_{1}-1\right) \geq \alpha$ for all $T \geq T^{*}$. This implies 46. 
Theorem 3.2 (Suboptimality). Let $y_{0} \in L^{2}(0, L)$ and a sampling time $\delta>0$ be given. Then there exist numbers, $T^{*}=T^{*}\left(\left\|y_{0}\right\|_{L^{2}(0, L)}, \delta\right)>\delta$, and $\alpha=\alpha\left(\left\|y_{0}\right\|_{L^{2}(0, L)}, \delta\right) \in(0,1)$, such that for every fixed prediction horizon $T \geq T^{*}$, the model predictive control $u_{m p c}$ obtained from Algorithm 1 satisfies

$$
\alpha V_{\infty}\left(y_{0}\right) \leq \alpha J_{\infty}\left(u_{m p c}, y_{0}\right) \leq V_{T}\left(y_{0}\right) \leq V_{\infty}\left(y_{0}\right) .
$$

Proof. The right and left inequalities are obvious, therefore we only need to verify the middle one.

First we show that $V_{T}\left(y_{0}\right)$ is bounded by a constant $r_{y_{0}}$ independent of $T$. We reconsider the proof of Lemma 3.2 to find

$$
V_{T}\left(y_{0}\right) \leq \gamma\left(T,\left\|y_{0}\right\|_{L^{2}(0, L)}\right)\left\|y_{0}\right\|_{L^{2}(0, L)}^{2} \leq \frac{(1+\beta) c\left(\left\|y_{0}\right\|_{L^{2}(0, L)}\right)}{2 \alpha_{\ell} \mu\left(\left\|y_{0}\right\|_{L^{2}(0, L)}\right)}\left\|y_{0}\right\|_{L^{2}(0, L)}^{2}=: r_{y_{0}}^{2} .
$$

Next we define the radius

$$
r_{0}:=\max \left\{\left\|y_{0}\right\|_{L^{2}(0, L)}, \sqrt{\frac{c_{\delta}}{\alpha_{\ell}} r_{y_{0}}^{2}}\right\},
$$

where the constant $c_{\delta}$, defined in Lemma 3.1, depends only on $\delta$.

For $d_{1}\left(r_{0}\right)$ defined as in Lemma 3.3 , due to Proposition 3.1. there exist positive numbers $T^{*}=$ $T^{*}\left(d_{1}, \delta\right)>\delta$, and $\alpha=\alpha\left(d_{1}, \delta\right) \in(0,1)$ such that the inequality 46 holds for every $T \geq T^{*}$ and $y_{0} \in \mathcal{B}_{r_{0}}(0)$. Therefore, in order to use the dissipative inequality 46 for every optimal solution pair $\left(y_{T}^{*}\left(\cdot ; y_{m p c}\left(t_{k}\right), t_{k}\right), u_{T}^{*}\left(\cdot ; y_{m p c}\left(t_{k}\right), t_{k}\right)\right)$ of Algorithm 1. we need to be sure, a priori, that

$$
y_{m p c}\left(t_{k}\right) \in \mathcal{B}_{r_{0}}(0) \quad \text { for every } k \in \mathbb{N}_{0} .
$$

We proceed by induction with respect to the sampling index $k$. For every $k \in \mathbb{N}_{0}$ we will show that the inequality

$$
V_{T}\left(y_{m p c}\left(t_{k}\right)\right) \leq V_{T}\left(y_{0}\right)-\alpha \int_{0}^{t_{k}} \ell\left(y_{m p c}(t), u_{m p c}(t)\right) d t
$$

and condition 50 hold true.

First, since $\left\|y_{0}\right\|_{L^{2}(0, L)} \leq r_{0}$, by Proposition 3.1 for every fixed $T \geq T^{*}\left(d_{1}, \delta\right)$ we have

$$
V_{T}\left(y_{m p c}\left(t_{1}\right)\right) \leq V_{T}\left(y_{0}\right)-\alpha \int_{0}^{t_{1}} \ell\left(y_{m p c}(t), u_{m p c}(t)\right) d t
$$

with an $\alpha=\alpha\left(d_{1}, \delta\right) \in(0,1)$. Moreover by using estimate $(30)$ we can infer that

$$
\begin{aligned}
\left\|y_{m p c}\left(t_{1}\right)\right\|_{L^{2}(0, L)}^{2} & \stackrel{30}{\leq} c_{\delta}\left(\int_{0}^{t_{1}}\left\|y_{m p c}(t)\right\|_{L^{2}(0, L)}^{2}+\left\|u_{m p c}(t)\right\|_{L^{2}(\Omega)}^{2}\right) d t \\
& \stackrel{3}{\leq} \frac{c_{\delta}}{\alpha_{\ell}} \int_{0}^{t_{1}} \ell\left(y_{m p c}(t), u_{m p c}(t)\right) d t \leq \frac{c_{\delta}}{\alpha_{\ell}} V_{T}\left(y_{0}\right) \stackrel{\text { 48 }}{\leq} \frac{c_{\delta}}{\alpha_{\ell}} r_{y_{0}}^{2} \stackrel{49 p}{\leq} r_{0}^{2} .
\end{aligned}
$$

Now to carry out the induction step, we assume that

$$
y_{m p c}\left(t_{k}\right) \in \mathcal{B}_{r_{0}}(0) \quad \text { for all } k=0, \ldots, k^{\prime},
$$

and that

for $k^{\prime} \in \mathbb{N}$.

$$
V_{T}\left(y_{m p c}\left(t_{k^{\prime}}\right)\right) \leq V_{T}\left(y_{0}\right)-\alpha \int_{0}^{t_{k^{\prime}}} \ell\left(y_{m p c}(t), u_{m p c}(t)\right) d t
$$

Since $y_{m p c}\left(t_{k^{\prime}}\right) \in \mathcal{B}_{r_{0}}(0)$, by Proposition 3.1 we have

$$
V_{T}\left(y_{m p c}\left(t_{k^{\prime}+1}\right)\right) \leq V_{T}\left(y_{m p c}\left(t_{k^{\prime}}\right)\right)-\alpha \int_{t_{k^{\prime}}}^{t_{k^{\prime}+1}} \ell\left(y_{m p c}(t), u_{m p c}(t)\right) d t .
$$


Combined with (54) this gives

$$
V_{T}\left(y_{m p c}\left(t_{k^{\prime}+1}\right)\right) \leq V_{T}\left(y_{0}\right)-\alpha \int_{0}^{t_{k^{\prime}+1}} \ell\left(y_{m p c}(t), u_{m p c}(t)\right) d t
$$

Moreover, by the same argument as in 52 we obtain

$$
\begin{aligned}
\left\|y_{m p c}\left(t_{k^{\prime}+1}\right)\right\|_{L^{2}(0, L)}^{2} & \stackrel{30}{\leq} c_{\delta} \int_{t_{k^{\prime}}}^{t_{k^{\prime}+1}}\left(\left\|y_{m p c}(t)\right\|_{L^{2}(0, L)}^{2}+\left\|u_{m p c}(t)\right\|_{L^{2}(\hat{\Omega})}^{2}\right) d t \\
& \leq \frac{3}{\alpha_{\ell}} \int_{t_{k^{\prime}}}^{t_{k^{\prime}+1}} \ell\left(y_{m p c}(t), u_{m p c}(t)\right) d t \\
& \leq \frac{c_{\delta}}{\alpha_{\ell}} V_{T}\left(y_{m p c}\left(t_{k^{\prime}}\right)\right) \stackrel{54}{\leq} \frac{c_{\delta}}{\alpha_{\ell}} V_{T}\left(y_{0}\right) \leq \frac{c_{\delta}}{\alpha_{\ell}} r_{y_{0}}^{2} \leq r_{0}^{2} .
\end{aligned}
$$

Hence $y_{m p c}\left(t_{k^{\prime}+1}\right) \in \mathcal{B}_{r_{0}}(0)$, which concludes the induction step. Taking the limit $k^{\prime} \rightarrow \infty$ we find

$$
\alpha J_{\infty}\left(u_{m p c}(\cdot), y_{0}\right)=\alpha \int_{0}^{\infty} \ell\left(y_{m p c}(t), u_{m p c}(t)\right) d t \leq V_{T}\left(y_{0}\right),
$$

which concludes the proof. Note that the constants $\alpha$ and $T^{*}$ depend only on $\delta$ and $\left\|y_{0}\right\|_{L^{2}(0, L)}$.

Theorem 3.3 (Exponential decay). Suppose that $y_{0} \in L^{2}(0, L)$ and let a sampling time $\delta>0$ be given. Then there exist numbers $T^{*}\left(\left\|y_{0}\right\|_{L^{2}(0, L)}, \delta\right)>\delta, \alpha\left(\left\|y_{0}\right\|_{L^{2}(0, L)}, \delta\right) \in(0,1)$ such that for every prediction horizon $T \geq T^{*}$, the model predictive trajectory $y_{m p c}(\cdot)$ satisfies

$$
V_{T}\left(y_{m p c}\left(t_{k}\right)\right) \leq e^{-\zeta t_{k}} V_{T}\left(y_{0}\right),
$$

where $\zeta$ is a positive number depending on $y_{0}, \delta$, and $T$. Moreover, for every positive $t$ we have

$$
\left\|y_{m p c}(t)\right\|_{L^{2}(0, L)}^{2} \leq c e^{-\zeta t}\left\|y_{0}\right\|_{L^{2}(0, L)}^{2}
$$

with a positive constant $c$ depending on $y_{0}, \delta$, and $T$.

Proof. Let $y_{0} \in L^{2}(0, L)$ and $\delta>0$ be given. Then according to Theorem 3.2, there exist positive numbers $T^{*}\left(\left\|y_{0}\right\|_{L^{2}(0, L)}, \delta\right)$ and $\alpha\left(\left\|y_{0}\right\|_{L^{2}(0, L)}, \delta\right)$ such that for every $T \geq T^{*}$ we have

$$
y_{m p c}\left(t_{k}\right) \in \mathcal{B}_{r_{0}}(0) \quad \text { for all } k \in \mathbb{N}_{0}
$$

with $r_{0}\left(\left\|y_{0}\right\|_{L^{2}(0, L)}\right)$ defined in Theorem 3.2 by 449$)$, and

$$
V_{T}\left(y_{m p c}\left(t_{k+1}\right)\right)-V_{T}\left(y_{m p c}\left(t_{k}\right)\right) \leq-\alpha \int_{t_{k}}^{t_{k+1}} \ell\left(y_{m p c}(t), u_{m p c}(t)\right) d t \quad \text { for every } k \in \mathbb{N} .
$$

By using (40) and 41, we have

$$
\begin{aligned}
V_{T}\left(y_{m p c}\left(t_{k+1}\right)\right) & \leq \theta_{1} \int_{t_{k+1}}^{t_{k}+T} \ell\left(y_{T}^{*}\left(t ; y_{m p c}\left(t_{k}\right), t_{k}\right), u_{T}^{*}\left(t ; y_{m p c}\left(t_{k}\right), t_{k}\right)\right) d t \\
& \leq \theta_{1} \theta_{2} \int_{t_{k}}^{t_{k+1}} \ell\left(y_{T}^{*}\left(t ; y_{m p c}\left(t_{k}\right), t_{k}\right), u_{T}^{*}\left(t ; y_{m p c}\left(t_{k}\right), t_{k}\right)\right) d t \\
& =\theta_{1} \theta_{2} \int_{t_{k}}^{t_{k+1}} \ell\left(y_{m p c}(t), u_{m p c}(t)\right) d t
\end{aligned}
$$


where $\theta_{1}=\theta_{1}\left(\delta, T, d_{1}\left(r_{0}\right)\right)>0$ and $\theta_{2}=\theta_{2}\left(\delta, T, d_{1}\left(r_{0}\right)\right)>0$ are defined in the statement of Lemma 3.4 and $d_{1}=d_{1}\left(r_{0}\right)$ is introduced by Lemma 3.3 . Now by combining (57) and (58) we obtain

$$
V_{T}\left(y_{m p c}\left(t_{k+1}\right)\right)-V_{T}\left(y_{m p c}\left(t_{k}\right)\right) \leq \frac{-\alpha}{\theta_{1} \theta_{2}} V_{T}\left(y_{m p c}\left(t_{k+1}\right)\right) \quad \text { for every } k \in \mathbb{N} .
$$

Therefore, by defining $\eta:=\left(1+\frac{\alpha}{\theta_{1} \theta_{2}}\right)^{-1}$ for every $k \in \mathbb{N}$ we can write

$$
V_{T}\left(y_{m p c}\left(t_{k}\right)\right) \leq \eta V_{T}\left(y_{m p c}\left(t_{k-1}\right)\right) \leq \eta^{2} V_{T}\left(y_{m p c}\left(t_{k-2}\right)\right) \leq \cdots \leq \eta^{k} V_{T}\left(y_{0}\right) .
$$

Defining $\zeta:=\frac{|\ln \eta|}{\delta}$, we obtain inequality $(55)$.

Turning to inequality (56), let $t>0$ be arbitrary. Then there exists an index $k$ such that $t \in\left[t_{k}, t_{k+1}\right]$. By using estimate (29) for the initial function $y_{m p c}\left(t_{k}\right)$, we have for $t \in\left[t_{k}, t_{k+1}\right]$

$$
\begin{aligned}
\left\|y_{m p c}(t)\right\|_{L^{2}(0, L)}^{2} & \leq\left\|y_{m p c}\left(t_{k}\right)\right\|_{L^{2}(0, L)}^{2}+\int_{t_{k}}^{t}\left(\left\|y_{m p c}(s)\right\|_{L^{2}(0, L)}^{2}+\left\|u_{m p c}(s)\right\|_{L^{2}(\hat{\Omega})}^{2}\right) d s \\
& \leq\left\|y_{m p c}\left(t_{k}\right)\right\|_{L^{2}(0, L)}^{2}+\frac{1}{\alpha_{\ell}} \int_{t_{k}}^{t} \ell\left(y_{m p c}(s), u_{m p c}(s)\right) d s \\
& \leq\left\|y_{m p c}\left(t_{k}\right)\right\|_{L^{2}(0, L)}^{2}+\frac{1}{\alpha_{\ell}} V_{T}\left(y_{m p c}\left(t_{k}\right)\right) .
\end{aligned}
$$

Moreover, by using estimate 30 we infer that

$$
\begin{aligned}
\left\|y_{m p c}\left(t_{k}\right)\right\|_{L^{2}(0, L)}^{2} & \leq c_{\delta} \int_{t_{k-1}}^{t_{k}}\left(\left\|y_{m p c}(t)\right\|_{L^{2}(0, L)}^{2}+\left\|u_{m p c}(t)\right\|_{L^{2}(\hat{\Omega})}^{2}\right) d t \\
& \leq \frac{c_{\delta}}{\alpha_{\ell}} \int_{t_{k-1}}^{t_{k}} \ell\left(y_{m p c}(t), u_{m p c}(t)\right) d t \leq \frac{c_{\delta}}{\alpha_{\ell}} V_{T}\left(y_{m p c}\left(t_{k-1}\right)\right) .
\end{aligned}
$$

By using (59), 60) and (61) we obtain for $t \in\left[t_{k}, t_{k+1}\right]$

$$
\begin{aligned}
\left\|y_{m p c}(t)\right\|_{L^{2}(0, L)}^{2} & \leq\left\|y_{m p c}\left(t_{k}\right)\right\|_{L^{2}(0, L)}^{2}+\frac{1}{\alpha_{\ell}} V_{T}\left(y_{m p c}\left(t_{k}\right)\right) \leq \frac{1+c_{\delta}}{\alpha_{\ell}} V_{T}\left(y_{m p c}\left(t_{k-1}\right)\right) \\
& \leq \frac{\left(1+c_{\delta}\right) \eta^{k+1}}{\alpha_{\ell} \eta^{2}} V_{T}\left(y_{0}\right) \leq \frac{1+c_{\delta}}{\alpha_{\ell} \eta^{2}} e^{-\zeta t_{k+1}} V_{T}\left(y_{0}\right) \\
& \leq \frac{1+c_{\delta}}{\alpha_{\ell} \eta^{2}} e^{-\zeta t} V_{T}\left(y_{0}\right) \leq \frac{\left(1+c_{\delta}\right) \gamma\left(T,\left\|y_{0}\right\|_{L^{2}(0, L)}\right)}{\alpha_{\ell} \eta^{2}} e^{-\zeta t}\left\|y_{0}\right\|_{L^{2}(0, L)}^{2}
\end{aligned}
$$

Setting $c:=\frac{\left(1+c_{\delta}\right) \gamma\left(T,\left\|y_{0}\right\|_{L^{2}(0, L)}\right)}{\alpha_{\ell} \eta^{2}}$, we conclude the proof.

\section{Discretization And NUMERical RESUlts}

This section is devoted to illustrating the MPC technique for stabilizing the KdV equaiton. We describe the discretization of the optimization problem (1)-(3), as well as the numerical optimization process we use. In the case of bounded domains numerous schemes for solving nonlinear KdV are available including finite differences 22, 57], finite elements [3, 55], finite volumes [23], discontinuous Galerkin schemes [8, 56], or polynomial spectral methods [41,42,53]. Spectral discretizations present interesting advantages regarding precision and simulation speed compared to any finite difference or finite element method [13]. 


\subsection{Discretization}

One of the most recent and efficient numerical methods for solving the Korteweg-de Vries equation with Dirichlet boundary conditions is proposed in 41]. The linear term is treated by a Petrov-Galerkin method based on Legendre polynomials, while the nonlinear term is treated pseudospectrally on the Chebyschev collocation points. Shortly after, Shen 53 proposed an improvement of this Petrov-Galerkin method with nearly optimal computational complexity. This will be our method of choice and we briefly recall it here.

\subsubsection{The dual Petrov-Galerkin method}

The test and trial function bases are chosen as a compact combination of Legendre polynomials in such a way that the trial functions satisfy the underlying boundary conditions of the primal equation and the test functions satisfy the boundary conditions as defined in (5). As a consequence, all matrices involved in the resolution of the problem are sparse 53. We present the method for the reference domain $\Omega:=(-1,1)$, but it can be extended to any other domain of the type $(a, b)$ by scaling the Legendre polynomials and the integrals. We denote by $P_{N}$ the space of polynomials of degree $\leq N$ and set

$$
\begin{gathered}
V_{N}=\left\{y \in P_{N}: y(1)=y(-1)=\partial_{x} y(1)=0\right\}, \\
V_{N}^{*}=\left\{y \in P_{N}: y(1)=y(-1)=\partial_{x} y(-1)=0\right\} .
\end{gathered}
$$

Then for $T>0$, we consider the semi-discrete problem: find

$$
y_{N}:[0, T] \rightarrow V_{N}, \quad t \mapsto y_{N}(t, \cdot),
$$

such that for almost every $t \in[0, T]$

$$
\left\langle\partial_{t} y_{N}, \varphi_{N}\right\rangle+\left(\partial_{x} y_{N}, \varphi_{N}\right)+\left(\partial_{x} y_{N}, \partial_{x x} \varphi_{N}\right)-\left(\frac{y_{N}^{2}}{2}, \partial_{x} \varphi_{N}\right)=\left(\chi_{\hat{\Omega}} u, \varphi_{N}\right) \quad \forall v_{N} \in V_{N}^{*}
$$

where $(\cdot, \cdot)$ denotes the usual $L^{2}(\Omega)$ spatial inner product, $\langle\cdot, \cdot\rangle$ is the spatial duality pairing between $H^{-2}(\Omega)$ and $H_{0}^{2}(\Omega)$, and $\hat{\Omega} \subseteq \Omega$ is the control domain, as in the continuous case.

Denoting by $L_{k}$ the $k$ th Legendre polynomial, the basis functions are defined as follows (see Figure 1)

$$
\begin{aligned}
& \phi_{k}(x)=L_{k}(x)-\frac{2 k+3}{2 k+5} L_{k+1}(x)-L_{k+2}(x)+\frac{2 k+3}{2 k+5} L_{k+3}(x), \\
& \psi_{k}(x)=L_{k}(x)+\frac{2 k+3}{2 k+5} L_{k+1}(x)-L_{k+2}(x)-\frac{2 k+3}{2 k+5} L_{k+3}(x) .
\end{aligned}
$$
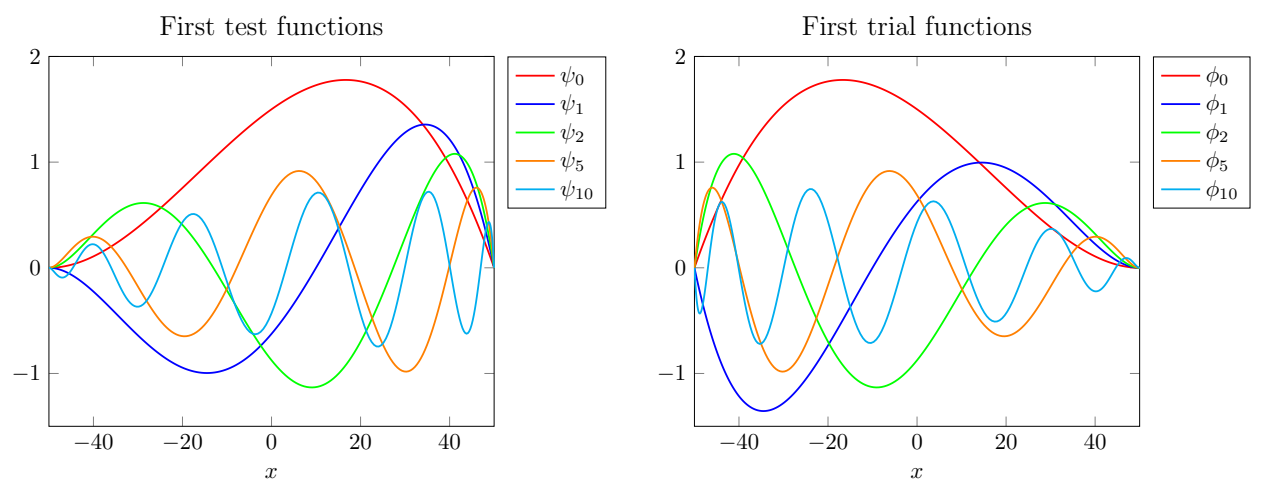

Figure 1. First test and trial functions. 
Thus for $N \geq 3$, we have

$$
V_{N}=\operatorname{span}\left\{\phi_{0}, \phi_{1}, \ldots, \phi_{N-3}\right\}, \quad V_{N}^{*}=\operatorname{span}\left\{\psi_{0}, \psi_{1}, \ldots, \psi_{N-3}\right\} .
$$

The semi-discrete state variable $y_{N}(t, \cdot)$ on the spectral space is given in vector representation as

$$
y_{N}(t, \cdot)=\sum_{k=0}^{N-3} \hat{y}_{k}(t) \phi_{k}(\cdot), \quad \mathbf{y}(t)=\left(\hat{y}_{0}(t), \hat{y}_{1}(t), \ldots, \hat{y}_{N-3}(t)\right)^{T} .
$$

Analogously the vector representation of the control is given by:

$$
\mathbf{u}(t)=\left(\left(u_{N}(t, \cdot), \psi_{0}(\cdot)\right),\left(u_{N}(t, \cdot), \psi_{1}(\cdot)\right), \ldots,\left(u_{N}(t, \cdot), \psi_{N-3}(\cdot)\right)\right)^{T},
$$

where the expression for the semi-discrete control $u_{N}(t, \cdot)$ is given in Section 4.1.2. Afterwards, one builds the matrices $\mathbf{M}, \mathbf{P}$, and $\mathbf{S}$ of size $(N-2) \times(N-2)$ with coefficients $m_{i j}, p_{i j}, q_{i j}$, and $s_{i j}$ defined as follows:

$$
m_{i j}=\left(\phi_{j}, \psi_{i}\right), \quad p_{i j}=\left(\partial_{x} \phi_{j}, \psi_{i}\right), \quad s_{i j}=\left(\partial_{x} \phi_{j}, \partial_{x x} \psi_{i}\right) .
$$

The variational formulation 62 thus yields

$$
\mathbf{M} \frac{d \mathbf{y}}{d t}+(\mathbf{P}+\mathbf{S}) \mathbf{y}+F(\mathbf{y})=\mathbf{B u}
$$

where $\mathbf{B}$ is the matrix representing the characteristic function $\chi_{\hat{\Omega}}$ in 62 and $F(\mathbf{y})$ represents the nonlinear term. It is approximated as suggested in 53] using the pseudospectral approach. Thus the nonlinearity is evaluated at the chosen Chebyshev-Gauss-Lobatto (CGL) points in the spatial domain and then it is transformed back to the Legendre spectral space in the efficient manner.

\subsubsection{Discretization of the control}

The control is discretized in space with piecewise linear, continuous finite elements on a grid whose nodes are the Chebyschev-Gauss-Lobatto points $\left(x_{n}\right), n=0, \ldots, N$ as previously mentioned. The various norms involved in the optimization problem are computed using the trapezoidal rule for the evaluation of the spatial integrals for each cell. Thus, $u_{N}=\sum_{j=1}^{N_{T}} \chi_{I_{j}} \sum_{n=0}^{N} \hat{\mathbf{u}}_{j n} e_{n}$, where $I_{j}=\left(\delta_{j-1}^{t}, \delta_{j}^{t}\right]$ is the $j^{t h}$ time interval corresponding to the grids $0=\delta_{0}^{t}<\delta_{1}^{t}<\cdots<\delta_{N_{T}}^{t}=T$. Moreover $e_{n}$ is the basis vector for piecewise linear, continuous finite elements centered at the grid point $x_{n}$. Then for $u_{N}$ it holds that

$$
\left\|u_{N}\right\|_{L^{2}\left(0, T ; L^{2}(\hat{\Omega})\right)}^{2}=\sum_{j=1}^{N_{T}} \Delta t\left(\sum_{n=0}^{N} d_{n} \hat{\mathbf{u}}_{n j}^{2}\right), \quad\left(u_{N}, \psi\right)=\sum_{j=1}^{N_{T}} \chi_{I_{j}} \sum_{n=0}^{N} d_{n} \hat{\mathbf{u}}_{j n} \psi_{n}
$$

for all spectral basis test functions $\psi$ where we have denoted $\psi_{n}=\psi\left(x_{n}\right)$, and $d_{n}=\int_{\hat{\Omega}} e_{n} \mathrm{~d} x$.

\subsubsection{Time-stepping scheme}

Following the idea in [11, 41, 42, 53, we use the multistep Crank-Nicolson Leap Frog scheme. In this setting, the third derivative is treated implicitely and the nonlinear term is treated explicitely. This allows to circumvent possible step size restrictions due to the third order derivative. In addtion, since the nonlinear term is treated explicitly, there is no need to solve a nonlinear system of equations at every time step. A proper derivation of the discrete adjoint and gradient is available in [11].

\subsection{Numerical examples}

In this section we present numerical experiments. They are based on Algorithm 2 that takes as initial input the time horizon $T_{\infty}$ and an initial condition $y_{0} \in L^{2}(\Omega)$. 


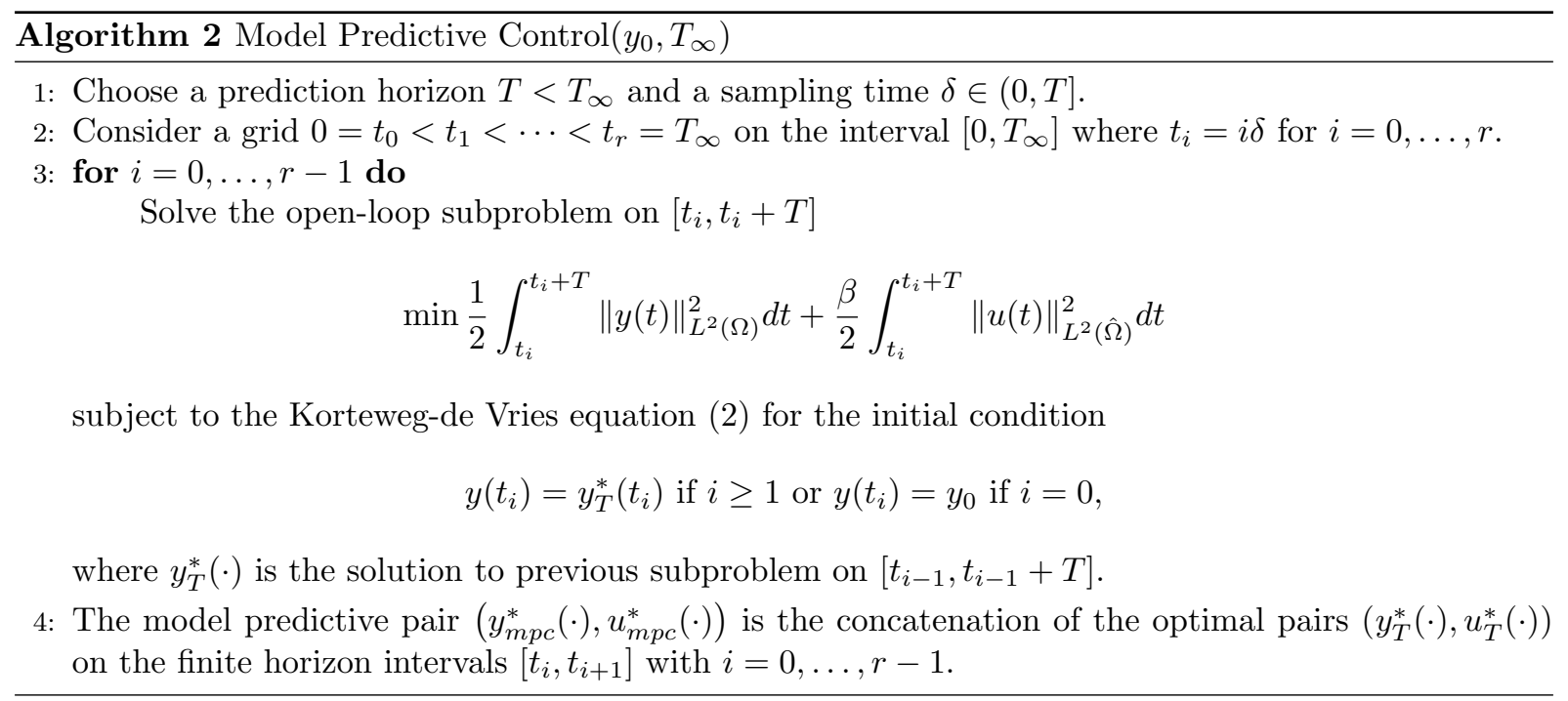

Each open-loop problem is solved with the help of Barzilai-Browein gradient steps 6 improved by a nonmonotonous line-search method [21]. Moreover we consider the following quantities in order to interpret the results of the stabilization problem for different settings:

(1) $J_{T_{\infty}}\left(u_{m p c}, y_{0}\right):=\frac{1}{2} \int_{0}^{T_{\infty}}\left\|y_{m p c}(t)\right\|_{L^{2}(\Omega)}^{2} d t+\frac{\beta}{2} \int_{0}^{T_{\infty}}\left\|u_{m p c}(t)\right\|_{L^{2}(\hat{\Omega})}^{2} d t$

(2) $\left\|y_{m p c}\right\|_{L^{2}(Q)}$ with $Q:=\left(0, T_{\infty}\right) \times \Omega$,

(3) $\left\|y_{m p c}\left(T_{\infty}\right)\right\|_{L^{2}(\Omega)}$

(4) iter : the total number of iterations (BB-gradient steps) that the optimizer needs for all open-loop problems on the intervals $\left(t_{i}, t_{i}+T\right)$ for $i=0, \ldots, r-1$.

Turning to the description of the numerical experiment that we carried out, we first recall that it is not known whether the system can be stabilized to zero without control, due to the fact that $\partial_{x} y(t, 0)$ might be zero for a domain with the critical lengths [20 49].

Here, we propose a situation where a soliton starts travelling at time $t=0$ (its initial shape is given in Figure 2(c) . On an infinite domain, a soliton is a solitary wave that travels at constant speed without losing its shape. This phenomenon is a result of the balance between nonlinearity and dispersion which typically occurs for the Korteweg-de Vries equation [19 38. In our case though, the initial soliton encounters the right boundary. Then its balance is broken and due to the dispersive effect, it is decomposed into several smaller reflected waves. See Figure 2(a) One of them evolves almost into a stationary one, while the other one travels at constant speed without hitting the boundaries. This is depicted in Figure 2(b) over a long period of time. In this case, i.e. without any control, the objective functional has the value $J_{T_{\infty}}=3152.8$, whilst $\|y\|_{L^{2}(Q)}=79.4$ and more importantly at the final time, $\left\|y\left(T_{\infty}\right)\right\|_{L^{2}(\Omega)}=4.9$.

As a very large time horizon is considered, this would be prohibitive to apply the classical open-loop control on problem (1)-(2). Hence, the use of model predictive control is key for stabilization. Our simulations are carried out with the choice of: $\Omega=(-10 \pi, 10 \pi), N=256, \beta=10^{-1}, \delta=1, T_{\infty}=200$, and various prediction horizons $T=1,1.5,2, y_{0}=12 \kappa^{2} \operatorname{sech}^{2}\left(\kappa\left(x-x_{0}\right)\right)$ with $\kappa=0.7$, and $x_{0}=0.0$. Finally, the control domain consists of two components and is given by

$$
\hat{\Omega}:=(-15.24,-8.00) \cup(7.74,15.14) .
$$

The results are gathered in Table 1 and Figure 3 - 6. In all three cases, the stabilizing measures are satisfying. As expected, the prediction horizon $T$ plays an important role. The smaller it is (i.e. the closer to the sampling time $\delta$ ), the fewer iterations are required (1098 for $T=1$ versus 1598 for $T=2$ ). However, one can observe from Figure 3 and Figure 4 - Figure 6, and it is verified by Table 1 , that a smaller time horizon leads to a less efficient, and slower stabilization. 


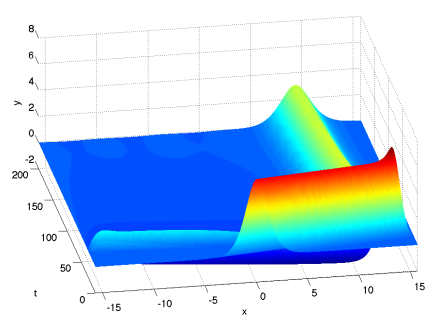

(a) State

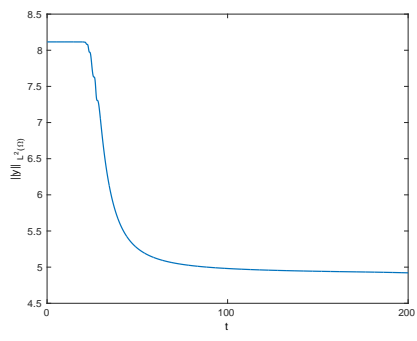

(b) Evolution of $L^{2}(0,1)$-norm of state

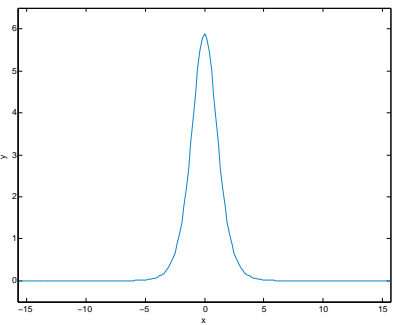

(c) State at $t=0$

Figure 2. Uncontrolled solution

\begin{tabular}{|c|c|c|c|c|}
\hline Prediction Horizon & $J_{T_{\infty}}$ & $\left\|y_{m p c}\right\|_{L^{2}(Q)}$ & $\left\|y_{m p c}\left(T_{\infty}\right)\right\|_{L^{2}(\Omega)}$ & iter \\
\hline$T=1.0$ & 1366 & 52.2 & $1.4 \times 10^{-5}$ & 1098 \\
\hline$T=1.5$ & 1051 & 45.7 & $6.4 \times 10^{-6}$ & 1386 \\
\hline$T=2.0$ & 728 & 37.8 & $4.1 \times 10^{-6}$ & 1598 \\
\hline
\end{tabular}

Table 1. Various indicators of the efficiency of the model predictive control process for different prediction horizons.

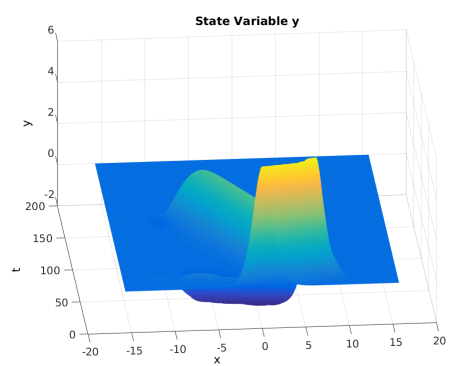

(a) $T=1$

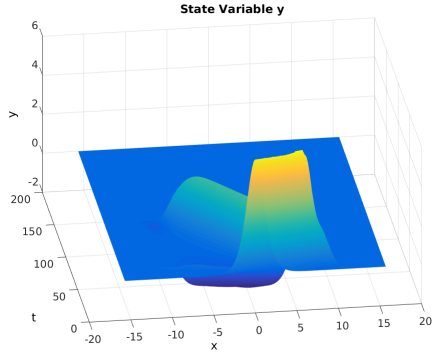

(b) $T=1.5$

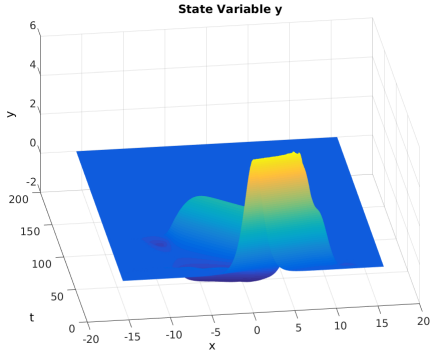

(c) $T=2$

Figure 3. Evolution of the state during the model predictive control process for the prediction horizons (from left to right): $T=1,1.5,2$.

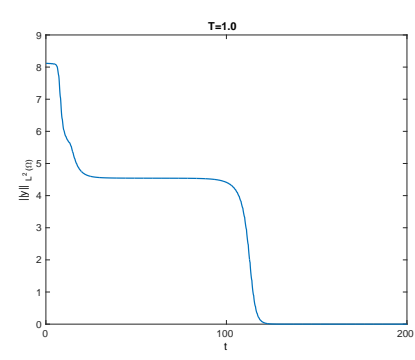

(a) $T=1$

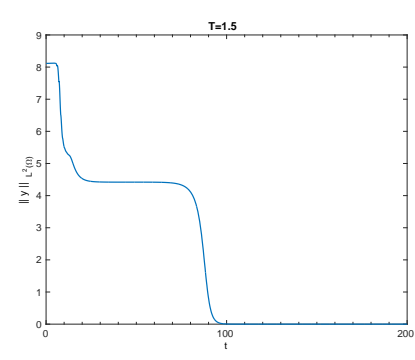

(b) $T=1.5$

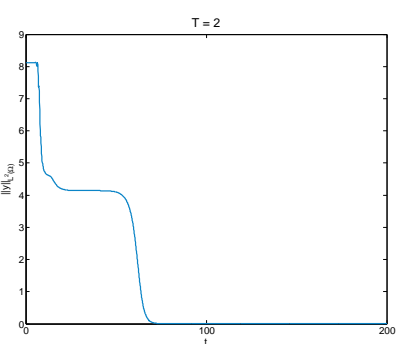

(c) $T=2$

Figure 4. Evolution of the $L^{2}$-norm of the state during the model predictive control process for the prediction horizons: $T=1,1.5,2$. 


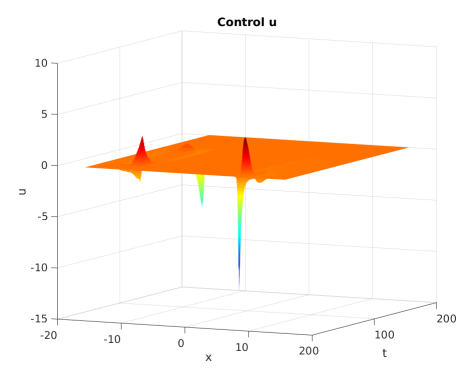

(a) $T=1$

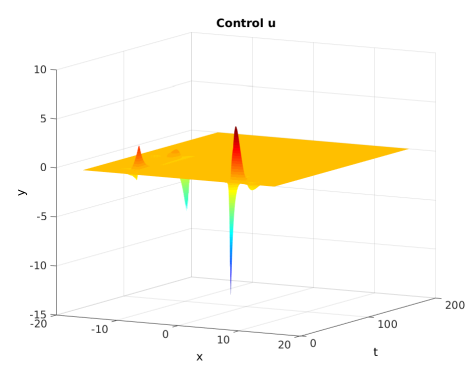

(b) $T=1.5$

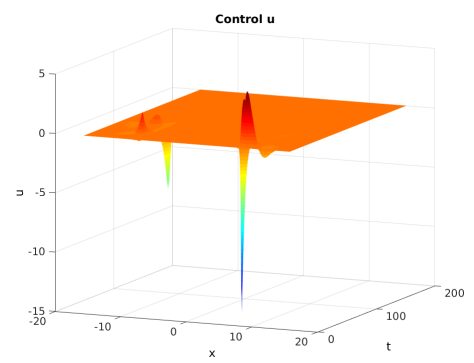

(c) $T=2$

Figure 5. Evolution of the control during the model predictive control process for the prediction horizons: $T=1,1.5,2$.

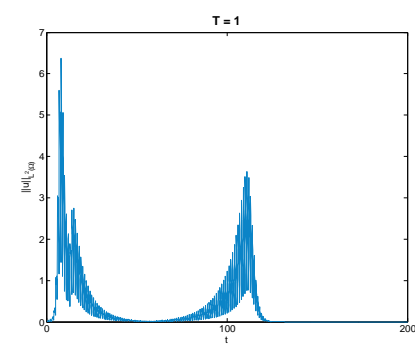

(a) $T=1$

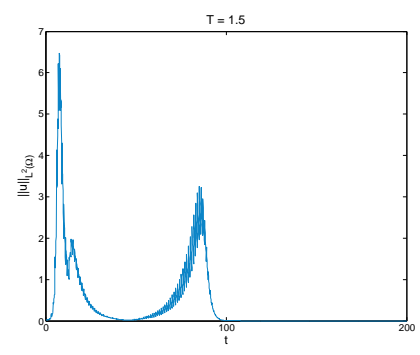

(b) $T=1.5$

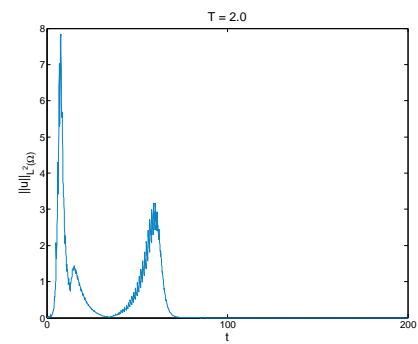

(c) $T=2$

Figure 6. Evolution of the $L^{2}$-norm of the control during the model predictive control process for the prediction horizons: $T=1,1.5,2$.

\section{Appendix A. Proofs of Lemmas 1.1 and 1.2}

\section{A.1. Proof of Lemma 1.1}

Assume that $y$ and $z \in \mathcal{B}_{0, T}$ are arbitrary. Then we have

$$
\begin{aligned}
\| y \partial_{x} y- & z \partial_{x} z\left\|_{L^{1}\left(0, T ; L^{2}(0, L)\right)} \leq\right\| y\left(\partial_{x} y-\partial_{x} z\right)\left\|_{L^{1}\left(0, T ; L^{2}(0, L)\right)}+\right\| \partial_{x} z(y-z) \|_{L^{1}\left(0, T ; L^{2}(0, L)\right)} \\
& \leq\left(\|y\|_{L^{2}\left(0, T ; L^{\infty}(0, L)\right)}\left\|\partial_{x} y-\partial_{x} z\right\|_{L^{2}\left(0, T ; L^{2}(0, L)\right)}+\left\|\partial_{x} z\right\|_{L^{2}\left(0, T ; L^{2}(0, L)\right)}\|y-z\|_{L^{2}\left(0, T ; L^{\infty}(0, L)\right)}\right) \\
& \leq C_{a}\|y\|_{L^{2}\left(0, T ; H_{0}^{1}(0, L)\right)}^{\frac{1}{2}}\|y\|_{L^{2}\left(0, T ; L^{2}(0, L)\right)}^{\frac{1}{2}}\|y-z\|_{L^{2}\left(0, T ; H_{0}^{1}(0, L)\right)} \\
& +C_{a}\|z\|_{L^{2}\left(0, T ; H_{0}^{1}(0, L)\right)}\|y-z\|_{L^{2}\left(0, T ; H_{0}^{1}(0, L)\right)}^{\frac{1}{2}}\|y-z\|_{L^{2}\left(0, T ; L^{2}(0, L)\right)}^{\frac{1}{2}}\|x\|_{L^{2}\left(0, T ; H_{0}^{1}(0, L)\right)} \\
& \left.\leq C_{a} T^{\frac{1}{4}}\|y\|_{L^{2}\left(0, T ; H_{0}^{1}(0, L)\right)}^{\frac{1}{2}} \| y(0, T] ; L^{2}(0, L)\right) \\
& +C_{a} T^{\frac{1}{4}}\|z\|_{L^{2}\left(0, T ; H_{0}^{1}(0, L)\right)}\|y-z\|_{L^{2}\left(0, T ; H_{0}^{1}(0, L)\right)}^{\frac{1}{2}}\|y-z\|_{C\left([0, T] ; L^{2}(0, L)\right)}^{\frac{1}{2}} \\
& =C_{a} T^{\frac{1}{4}}\left(\|y\|_{\mathcal{B}_{0, T}}+\|z\|_{\left.\mathcal{B}_{0, T}\right)}\right)\|y-z\|_{\mathcal{B}_{0, T}},
\end{aligned}
$$

where the constant $C_{a}$ stands for the Agmon's inequality. By taking $z=0$ in (8), we see that $y \partial_{x} y \in$ $L^{1}\left(0, T ; L^{2}(0, L)\right)$. 


\section{A.2. Proof of Lemma 1.2}

Multiplying (4) with $y$ and integrating on $(0, L)$ we have

$$
\begin{aligned}
\frac{1}{2} \frac{d}{d t}\|y(t)\|_{L^{2}(0, L)}^{2} & +\frac{1}{2}\left(\partial_{x} y(t, 0)\right)^{2}=\langle y(t), f(t)\rangle_{L^{2}} \\
& \leq\|y(t)\|_{L^{2}(0, L)}\|f(t)\|_{L^{2}(0, L)} \leq \frac{1}{2}\|y(t)\|_{L^{2}(0, L)}^{2}+\frac{1}{2}\|f(t)\|_{L^{2}(0, L)}^{2},
\end{aligned}
$$

where we assume that the solution $y$ is smooth enough to allow the calculations. Integrating on $\left(0, T^{\prime}\right)$ and using Gronwall's inequality we have

$$
\|y(t)\|_{L^{2}(0, L)}^{2} \leq \exp (T)\left(\left\|y_{0}\right\|_{L^{2}(0, L)}^{2}+\|f\|_{L^{2}\left(0, T ; L^{2}(0, L)\right)}^{2}\right), \quad \text { for all } t \in\left[0, T^{\prime}\right] .
$$

Now by a density argument and considering the fact that for $y_{0} \in \mathcal{D}(\mathcal{A})$ and $f \in C^{1}\left([0, T] ; L^{2}(0, L)\right)$, the solution $y$ of (4) belongs to the space $L^{2}\left(0, T^{\prime} ; H^{4}(0, L)\right) \cap C\left(\left[0, T^{\prime}\right] ; H^{3}(0, L)\right)$ 25, we can write

$$
|y|_{L^{\infty}\left(0, T^{\prime} ; L^{2}(0, L)\right)} \leq C_{1}\left(T, y_{0}, f\right),
$$

with

$$
C_{1}\left(T, y_{0}, f\right):=\left(\exp (T)\left(\left\|y_{0}\right\|_{L^{2}(0, L)}^{2}+\|f\|_{L^{2}\left(0, T ; L^{2}(0, L)\right)}^{2}\right)\right)^{\frac{1}{2}},
$$

for every $y_{0} \in L^{2}(0, L)$ and $f \in L^{2}\left(0, T, L^{2}(0, L)\right)$.

It remains to find an estimate for the term $\left\|\partial_{x} y\right\|_{L^{2}\left(0, T ; L^{2}(0, T)\right)}$. As in Lemma 1.1 we assume that the solution is smooth enough. Then by multiplying equation (4) by $x y$ and integrating over $(0, L)$, for every $t \in\left(0, T^{\prime}\right)$ we have

$$
\begin{aligned}
\frac{d}{d t} \int_{0}^{L}\left|x^{\frac{1}{2}} y(t, \cdot)\right|^{2} d x+3 \int_{0}^{L}\left(\partial_{x} y\right)^{2}(t, \cdot) d x & +x\left(\partial_{x} y\right)^{2}(t, 0) \\
& =\int_{0}^{L} y^{2}(t, \cdot) d x+\frac{2}{3} \int_{0}^{L} y^{3}(t, \cdot) d x+2 \int_{0}^{L} x f y d x .
\end{aligned}
$$

Moreover, we have for almost every $t \in\left(0, T^{\prime}\right)$

$$
\int_{0}^{L}|y(t, \cdot)|^{2} d x \leq\|y\|_{L^{\infty}\left(0, T^{\prime} ; L^{2}(0, L)\right)}^{2}
$$

and

$$
\begin{aligned}
\frac{2}{3} \int_{0}^{L}|y(t, \cdot)|^{3} d x \leq \frac{2}{3}\|y(t)\|_{L^{\infty}(0, L)}\|y\|_{L^{\infty}\left(0, T^{\prime} ; L^{2}(0, L)\right)}^{2} & \leq \frac{2 c^{\prime}}{3}\left\|\partial_{x} y(t)\right\|_{L^{2}(0, L)}\|y\|_{L^{\infty}\left(0, T^{\prime} ; L^{2}(0, L)\right)}^{2} \\
& \leq \frac{\epsilon c^{\prime}}{3}\left\|\partial_{x} y(t)\right\|_{L^{2}(0, L)}^{2}+\frac{c^{\prime}}{3 \epsilon}\|y\|_{L^{\infty}\left(0, T^{\prime} ; L^{2}(0, L)\right)}^{4},
\end{aligned}
$$

where the constant $c^{\prime}$ is the embedding constant of $H_{0}^{1}(0, L)$ into $L^{\infty}(0, L)$, and the positive number $\epsilon$ will be chosen later. Furthermore we have

$$
2 \int_{0}^{L}|x f y| d x \leq L\|f(t)\|_{L^{2}(0, L)}^{2}+L\|y(t)\|_{L^{2}(0, L)}^{2} \leq L\|f(t)\|_{L^{2}(0, L)}^{2}+L\|y\|_{L^{\infty}\left(0, T^{\prime} ; L^{2}(0, L)\right)}^{2} .
$$


Now by choosing $\epsilon:=\frac{6}{c^{\prime}}$, and combining inequalities $(73), 74$, and 75 with 72 , we obtain

$$
\begin{aligned}
\frac{d}{d t} \int_{0}^{L}\left|x^{\frac{1}{2}} y(t, \cdot)\right|^{2} d x & +\int_{0}^{L}\left(\partial_{x} y\right)^{2}(t, \cdot) d x \\
& \leq(1+L)\|y\|_{L^{\infty}\left(0, T^{\prime} ; L^{2}(0, L)\right)}^{2}+\frac{c^{\prime 2}}{18}\|y\|_{L^{\infty}\left(0, T^{\prime} ; L^{2}(0, L)\right)}^{4}+L\|f(t)\|_{L^{2}(0, L)}^{2}
\end{aligned}
$$

Integration with respect to $t$ over interval $\left(0, T^{\prime}\right)$ implies that

$$
\begin{aligned}
& \int_{0}^{L}\left|x^{\frac{1}{2}} y\left(T^{\prime}, \cdot\right)\right|^{2} d x+\int_{0}^{T^{\prime}} \int_{0}^{L}\left(\partial_{x} y\right)^{2} d x d t \\
& \quad \leq L\left\|y_{0}\right\|_{L^{2}(0, L)}^{2}+T(1+L)\|y\|_{L^{\infty}\left(0, T^{\prime} ; L^{2}(0, L)\right)}^{2}+T \frac{c^{\prime 2}}{18}\|y\|_{L^{\infty}\left(0, T^{\prime} ; L^{2}(0, L)\right)}^{4}+L\|f\|_{L^{2}\left(0, T ; L^{2}(0, L)\right)}^{2} \\
& \quad \leq L\left\|y_{0}\right\|_{L^{2}(0, L)}^{2}+T(1+L) C_{1}^{2}\left(T, y_{0}, f\right)+T \frac{c^{\prime 2}}{18} C_{1}^{4}\left(T, y_{0}, f\right)+L\|f\|_{L^{2}\left(0, T ; L^{2}(0, L)\right)}^{2}
\end{aligned}
$$

and as consequence of 70 and $(71)$, we can conclude that

$$
|y|_{\mathcal{B}_{0, T^{\prime}}} \leq K_{1}\left(T, L,\left|y_{0}\right|_{L^{2}(0, L)},|f|_{L^{2}\left(0, T ; L^{2}(0, L)\right)}\right)
$$

Turing to inequality (11), we obtain from $(78)$ that

$$
\begin{aligned}
& \left\|\partial_{t} y\right\|_{L^{2}\left(0, T^{\prime} ; H^{-2}(0, L)\right)}=\sup _{\|\phi\|_{L^{2}\left(0, T ; H_{0}^{2}(0, L)\right)}=1} \int_{0}^{T^{\prime}}\left\langle\partial_{t} y, \phi\right\rangle_{H^{-2}, H_{0}^{2}} \\
& \quad=\sup _{\|\phi\|_{\left.L^{2}\left(0, T^{\prime} ; H_{0}^{2}(0, L)\right)\right)}=1} \int_{0}^{L} \int_{0}^{T^{\prime}}\left(-\partial_{x} y \phi-\partial_{x} y \partial_{x}^{2} \phi-y \partial_{x} y \phi+f \phi\right) d x d t \\
& \leq\left(2+c_{1}\|y\|_{L^{\infty}\left(0, T^{\prime} ; L^{2}(0, L)\right)}\right)\|y\|_{L^{2}\left(0, T^{\prime} ; H_{0}^{1}(0, L)\right)}+\|f\|_{L^{2}\left(0, T ; L^{2}(0, L)\right)} \\
& \leq\left(2+c_{1} K_{1}\left(T, L,\left|y_{0}\right|_{L^{2}(0, L)},|f|_{L^{2}\left(0, T ; L^{2}(0, L)\right)}\right) K_{1}\left(T, L,\left|y_{0}\right|_{L^{2}(0, L)},|f|_{L^{2}\left(0, T ; L^{2}(0, L)\right)}\right)\right. \\
& \quad+\|f\|_{L^{2}\left(0, T ; L^{2}(0, L)\right)}
\end{aligned}
$$

where $c_{1}$ stands for the continuous embedding from $H^{2}(0, L)$ to $L^{\infty}(0, L)$. Combining $(78)$ and $(70)$ we conclude 11.

\section{REFERENCES}

[1] F. Allgöwer, T. A. Badgwell, J. S. Qin, J. B. Rawlings, And S. J. Wright, Nonlinear predictive control and moving horizon estimationan introductory overview, in Advances in control, Springer, 1999, pp. 391-449.

[2] F. Allgöwer and A. Zheng, Nonlinear model predictive control, vol. 26, Birkhäuser Basel, 2000.

[3] D. N. Arnold And R. Winther, A superconvergent finite element method for the Korteweg-de Vries equation, mathematics of computation, 38 (1982), pp. 23-36.

[4] B. Azmi And K. Kunisch, On the Stabilizability of the Burgers Equation by Receding Horizon Control, SIAM J. Control Optim., 54 (2016), pp. 1378-1405.

[5] J. BALL, Strongly continuous semigroups, weak solutions, and the variation of constants formula, Proceedings of the American Mathematical Society, 63 (1977), pp. 370-373.

[6] J. Barzilai And J. M. Borwein, Two-point step size gradient methods, IMA Journal of Numerical Analysis, 8 (1988), pp. $141-148$.

[7] A. Bensoussan, G. Da Prato, M. C. Delfour, and S. K. Mitter, Representation and control of infinite dimensional systems, Springer Science \& Business Media, 2007.

[8] J. L. Bona, V. A. Dougalis, and O. A. Karakashian, Fully discrete Galerkin methods for the Korteweg-de Vries equation, Computers and Mathematics with Applications, 12 (1986), pp. $859-884$.

[9] J. L. Bona, S.-M. Sun, And B.-Y. Zhang, A nonhomogeneous boundary-value problem for the Korteweg-de Vries equation posed on a finite domain, Comm. Partial Differential Equations, 28 (2003), pp. 1391-1436. 
[10] J. L. Bona, S. M. Sun, And B.-Y. Zhang, A non-homogeneous boundary-value problem for the Korteweg-de Vries equation posed on a finite domain II, Journal of Differential Equations, 247 (2009), pp. 2558-2596.

[11] A.-C. Boulanger and P. Trautmann, Sparse optimal control of the Korteweg-de Vries-Burgers equation on a bounded domain, Preprint, (2015).

[12] J. Boussinesq, Essai sur la théories des eaux courantes, Mémoires présentés par divers savants à l', Académic des Sciences de l'Institut Nationale de France, 23 (1877).

[13] J. P. Boyd, Chebyshev and Fourier spectral methods, Courier Corporation, 2001.

[14] H. Brezis, Functional analysis, Sobolev spaces and partial differential equations, Springer Science \& Business Media, 2010.

[15] R. A. Capistrano-Filho, A. F. Pazoto, and L. Rosier, Internal controllability of the Korteweg-de Vries equation on a bounded domain, ESAIM: Control, Optimisation and Calculus of Variations, 21 (2015), pp. 1076-1107.

[16] E. CERPA, Exact controllability of a nonlinear Korteweg-de Vries equation on a critical spatial domain, SIAM Journal on Control and Optimization, 46 (2007), pp. 877-899.

[17] H. Chen And F. Allgöwer, A quasi-infinite horizon nonlinear model predictive control scheme with guaranteed stability, Automatica, 34 (1998), pp. 1205-1217.

[18] T. Colin, J.-M. Ghidaglia, Et Al., An initial-boundary value problem for the Korteweg-de Vries equation posed on a finite interval, Advances in Differential Equations, 6 (2001), pp. 1463-1492.

[19] A. Constantin and R. Johnson, On the Non-Dimensionalisation, Scaling and Resulting Interpretation of the Classical Governing Equations for Water Waves, Journal of Nonlinear Mathematical Physics, 15 (2008), p. 5873.

[20] J.-M. Coron and E. Crépeau, Exact boundary controllability of a nonlinear KdV equation with critical lengths, J. Eur. Math. Soc. (JEMS), 6 (2004), pp. 367-398.

[21] Y.-H. Dai And H. Zhang, Adaptive two-point stepsize gradient algorithm, Numerical Algorithms, 27 (2001), pp. 377385.

[22] K. Djidjeli, W. G. Price, E. H. Twizell, and Y. Wang, Numerical methods for the solution of the third-and fifth-order dispersive Korteweg-de Vries equations, Journal of computational and applied mathematics, 58 (1995), pp. 307-336.

[23] D. Dutykh, T. Katsaounis, and D. Mitsotakis, Finite volume methods for unidirectional dispersive wave models, International Journal for Numerical Methods in Fluids, 71 (2013), pp. 717-736.

[24] A. Faminskit et AL., Global well-posedness of two initial-boundary-value problems for the Korteweg-de Vries equation, Differential and Integral Equations, 20 (2007), pp. 601-642.

[25] A. V. FAminski And N. A. LARKin, Initial-boundary value problems for quasilinear dispersive equations posed on a bounded interval, Electron. J. Differential Equations, 2010 (2010), pp. 1-20.

[26] O. Glass and S. Guerrero, Controllability of the Korteweg-de Vries equation from the right dirichlet boundary condition, Systems \& Control Letters, 59 (2010), pp. 390-395.

[27] G. Grimm, M. J. Messina, S. E. Tuna, And A. R. Teel, Model predictive control: for want of a local control Lyapunov function, all is not lost, Automatic Control, IEEE Transactions on, 50 (2005), pp. 546-558.

[28] L. Grüne, Analysis and design of unconstrained nonlinear MPC schemes for finite and infinite dimensional systems, SIAM Journal on Control and Optimization, 48 (2009), pp. 1206-1228.

[29] L. Grüne and J. Pannek, Nonlinear Model Predictive Control, Springer London, 2011.

[30] L. Grüne And A. Rantzer, On the infinite horizon performance of receding horizon controllers, Automatic Control, IEEE Transactions on, 53 (2008), pp. 2100-2111.

[31] J. Holmer, The initial-boundary value problem for the Korteweg-de Vries equation, Communications in Partial Differential Equations, 31 (2006), pp. 1151-1190.

[32] K. Ito AND K. Kunisch, Receding horizon optimal control for infinite dimensional systems, ESAIM: Control, Optimisation and Calculus of Variations, 8 (2002), pp. 741-760.

[33] — - Receding horizon control with incomplete observations, SIAM journal on control and optimization, 45 (2006), pp. 207-225.

[34] A. Jadbabaie And J. Hauser, On the stability of receding horizon control with a general terminal cost, Automatic Control, IEEE Transactions on, 50 (2005), pp. 674-678.

[35] A. Jadbabaie, J. YU, AND J. HaUser, Unconstrained receding-horizon control of nonlinear systems, Automatic Control, IEEE Transactions on, 46 (2001), pp. 776-783.

[36] C. JiA And B.-Y. Zhang, Boundary stabilization of the Korteweg-de Vries equation and the Korteweg-de Vries-Burgers equation, Acta applicandae mathematicae, 118 (2012), pp. 25-47.

[37] D. Kordeweg AND G. DE VRIES, On the change of form of long waves advancing in a rectangular channel, and a new type of long stationary wave, Phil. Mag, 39 (1895), pp. 422-443.

[38] D. J. Korteweg And G. DE VRIES, Xli. on the change of form of long waves advancing in a rectangular canal, and on a new type of long stationary waves, The London, Edinburgh, and Dublin Philosophical Magazine and Journal of Science, 39 (1895), p. 422443.

[39] E. F. Kramer and B. Zhang, Nonhomogeneous boundary value problems for the Korteweg-de Vries equation on a bounded domain, Journal of Systems Science and Complexity, 23 (2010), pp. 499-526.

[40] F. Linares And A. Pazoto, On the exponential decay of the critical generalized Korteweg-de Vries equation with localized damping, Proceedings of the American Mathematical Society, 135 (2007), pp. 1515-1522. 
[41] H. Ma And W. Sun, A Legendre-Petrov-Galerkin and Chebyshev collocation method for third-order differential equations, SIAM Journal on Numerical Analysis, 38 (2000), pp. 1425-1438.

[42] — Optimal error estimates of the Legendre-Petrov-Galerkin method for the Korteweg-de Vries equation, SIAM Journal on Numerical Analysis, 39 (2001), pp. 1380-1394.

[43] C. P. Massarolo, G. P. Menzala, and A. F. Pazoto, On the uniform decay for the Korteweg-de Vries equation with weak damping, Mathematical methods in the applied sciences, 30 (2007), pp. 1419-1435.

[44] D. Q. Mayne, J. B. Rawlings, C. V. Rao, and P. O. Scokaert, Constrained model predictive control: Stability and optimality, Automatica, 36 (2000), pp. 789-814.

[45] G. P. Menzala, C. F. Vasconcellos, and E. Zuazua, Stabilization of the Korteweg-de Vries equation with localized damping, Quarterly of Applied Mathematics, 60 (2002), pp. 111-129.

[46] A. F. PAzoto, Unique continuation and decay for the Korteweg-de Vries equation with localized damping, ESAIM: Control, Optimisation and Calculus of Variations, 11 (2005), pp. 473-486.

[47] A. PAZY, Semigroups of linear operators and applications to partial differential equations, vol. 44, Springer Science \& Business Media, 2012.

[48] M. Reble And F. Allgöwer, Unconstrained model predictive control and suboptimality estimates for nonlinear continuous-time systems, Automatica, 48 (2012), pp. 1812-1817.

[49] L. Rosier, Exact boundary controllability for the Korteweg-de Vries equation on a bounded domain, ESAIM: Control, Optimisation and Calculus of Variations, 2 (1997), pp. 33-55.

[50] - Control of the surface of a fluid by a wavemaker, ESAIM: Control, Optimisation and Calculus of Variations, 10 (2004), pp. 346-380.

[51] L. Rosier And B.-Y. Zhang, Global stabilization of the generalized Korteweg-de Vries equation posed on a finite domain, SIAM Journal on Control and Optimization, 45 (2006), pp. 927-956.

[52] _ Control and stabilization of the Korteweg-de Vries equation: recent progresses, Journal of Systems Science and Complexity, 22 (2009), pp. 647-682.

[53] J. Shen, A new dual-Petrov-Galerkin method for third and higher odd-order differential equations: application to the KDV equation, SIAM Journal on Numerical Analysis, 41 (2003), pp. 1595-1619.

[54] J. Simon, Compact sets in the space $L^{p}(0, T ; B)$, Ann. Mat. Pura Appl. (4), 146 (1987), pp. 65-96.

[55] R. Winther, A conservative finite element method for the Korteweg-de Vries equation, Mathematics of Computation, (1980), pp. 23-43.

[56] J. Yan And C.-W. Shu, A local discontinuous Galerkin method for KdV type equations, SIAM Journal on Numerical Analysis, 40 (2002), pp. 769-791.

[57] N. J. Zabusky AND M. D. KRuskal, Interaction of solitons in a collisionless plasma and the recurrence of initial states, Phys. Rev. Lett, 15 (1965), pp. 240-243.

[58] B.-Y. ZhAng, Exact boundary controllability of the Korteweg-de Vries equation, SIAM Journal on Control and Optimization, 37 (1999), pp. 543-565. 